\title{
The planar X-junction flow: stability analysis and control
}

\author{
IMA N L A S H G A R I ${ }^{1}$, \\ OUTI TAMMISOL A ${ }^{2} \dagger$, VINCENZO CITRO ${ }^{3}$, \\ MATTHEW P. JUNIPER AND LUCA BRANDT \\ ${ }^{1}$ Linné Flow Centre and SeRC (Swedish e-Science Research Centre), \\ KTH Mechanics, S-100 44 Stockholm, Sweden \\ ${ }^{2}$ DIIN, University of Salerno, via Ponte don Melillo, 84084 Fisciano (SA), Italy \\ ${ }^{3}$ Department of Engineering, University of Cambridge, Trumpington Street, \\ Cambridge CB2 1PZ, UK
}

(Received ?; revised ?; accepted ?)

The bifurcations and control of the flow in a planar X-junction are studied via linear stability analysis and direct numerical simulations. This study reveals the instability mechanisms in a symmetric channel junction and shows how these can be stabilized or destabilized by boundary modification. We observe two bifurcations as the Reynolds number increases. They both scale with the inlet speed of the two side channels and are almost independent of the inlet speed of the main channel. Equivalently, both bifurcations appear when the recirculation zone(s) reach a critical length. A two-dimensional stationary global mode becomes unstable first, changing the flow from a steady symmetric state to a steady asymmetric state via a pitchfork bifurcation. The core of this instability, whether defined by the structural sensitivity or the disturbance energy production, is at the edges of the recirculation bubbles, which are located symmetrically along the walls of the downstream channel. The energy analysis shows that the first bifurcation is due to a lift-up mechanism. We develop an adjustable control strategy for the first bifurcation with distributed suction/blowing at the walls. The linearly optimal wall-normal velocity distribution is computed through a sensitivity analysis and is shown to delay the first bifurcation from $R e=82.5$ to $R e=150$. This stabilizing effect arises because blowing at the walls weakens the streamwise velocity gradient around the recirculation zone and hinders the lift-up.At the second bifurcation, a three-dimensional stationary global mode with a span-wise wavenumber of order unity becomes unstable around the asymmetric steady state. Nonlinear three-dimensional simulations at the second bifurcation display transition to a nonlinear cycle involving growth of a three-dimensional steady structure, time-periodic secondary instability, and nonlinear breakdown restoring a two-dimensional flow. Finally, we show that the sensitivity to wall suction at the second bifurcation is as large as it is at the first bifurcation, providing a possible mechanism for destabilization.

Key words: Global instability, Flow focusing, X-junction, sensitivity analysis, control

$\dagger$ Email address for correspondence: olot2@cam.ac.uk 


\section{Introduction}

In this study, we examine the stability and control of the flow in a planar twodimensional X-junction, which comprises three inlet channels and one outlet channel. We employ three-dimensional direct numerical simulation (DNS) and global linear stability analysis to discover the nature of the first and second bifurcations. Finally we use adjoint-based sensitivity analysis to control the flow by applying apply optimal steady suction/blowing at the wall.

The present study is mostly fundamental in nature. The flow in the X-junction is a complex, wall-bounded, separated flow. Understanding the bifurcation mechanisms of this flow and designing control strategies to influence them is interesting in its own right, and adds to the physical understanding of a more general class of separated flows, in particular channel junctions and similar configurations (for example Oliveira et al. 2012; Poole et al. 2007; Chiang \& Sheu 2002; Lanzerstorfer \& Kuhlmann 2012). In the present manuscript we show that the critical parameter for the onset of both $2 \mathrm{D}$ and $3 \mathrm{D}$ instabilities of the $\mathrm{X}$-junction is the length of the recirculation bubble(s) rather than the Reynolds number. This indicates that similar instabilities can be found at lower or higher Reynolds flows that develop a similar topology to these recirculation zones.

$\mathrm{X}$-junctions exist in many natural phenomena, such as river junctions, and industrial applications, such as micromixers and flow focusing devices. Even though the typical Reynolds number in river engineering is higher than that in the present study (order of 1000-100000), the mean flow streamlines and the recirculation zone downstream of the junction are qualitatively similar (see e.g. Best 1987). The structure of the recirculation zone of a river is directly connected to sediment transport and accumulation (river bed formations) (Best \& Reid 1984; Shabayek et al. 2002). Conversely, the Reynolds number for micromixers and flow focusers is often low $(\operatorname{Re}<10)$, except for inertial micromixers (at $\mathrm{Re}=100-1000$ ), where the formation of the recirculation zone downstream of the junction is evident even without non-Newtonian effects.

Micromixers are designed to mix two fluids as efficiently as possible at small length scales, most often at low Reynolds numbers and in the absence of turbulence (Nguyen $\& \mathrm{Wu} 2005$ ). Flow focusers are often used to produce controlled-size droplets (Joanicot \& Ajdari 2005), and have been tested for the purpose of creating thread-like fibres in materials processing (Kinahan et al. 2011; Håkansson 2012). In the present work we propose a control strategy by fluid injection/removal (steady suction/blowing), which could be applicable for recirculatory flows in mixers and focusers despite the low Reynolds number.

Both mixing and focusing of the flow in $\mathrm{X}$ - and T-shaped junctions have been investigated in previous studies. However, there have only been a few studies in the area of inertia-driven hydrodynamic instabilities. Oliveira, Pinho \& Alves (2012) numerically and experimentally studied the vortical structure of a Newtonian fluid through an Xjunction. Their numerical study was two-dimensional. They defined the velocity ratio, $V_{r}$, as the ratio of the maximum velocity in the side channel to that in the main channel, and the width ratio, $W_{r}$, similarly. They found that the critical Reynolds number, based on the exit channel quantities, is about $R e \approx 140$ for $V_{r}=50$. They showed that the instabilities take the form of central vortices in the exit channel.

Mixers have been studied in more detail. Flow regimes in a T-mixer with a rectangular cross section are reported in the numerical study by Kockmann et al. (2003). As they increased the Reynolds number, they observed laminar flow, then vortical flow, and then engulfment flow, in which the streamlines in the mixing channel are asymmetric. Tomas, Ameel \& Guilkey (2010) experimentally studied a Newtonian fluid within a T- 
junction with a rectangular cross section. They observed that the flow first bifurcates to a steady asymmetric state at Reynolds number, $R e=105$, and then a highly oscillatory asymmetric flow occurs in the range $190<R e<240$. At higher Re, the flow regains its symmetric state and the rate of mixing decreases. Recently, Fani et al. (2013) studied the engulfment regime in a three-dimensional T-mixer with a quadratic cross-section by direct numerical simulation and a global stability analysis. The mechanism of engulfment was found to be the tilting of the vortical structures at the beginning of the exit channel. They also studied the sensitivity to the perturbation inlet velocity and reported that the flow with non-fully-developed inflow condition tends to be more stable.

Other studies in planar X-junction geometries, (Poole et al. 2007, 2014) considered rheology measurements. These authors recently extended their studies to the inertial regime (Poole et al. 2014). In their DNS of Newtonian inertial fluids on the same twodimensional configuration as ours, but with a different inflow-outflow structure, they find a very similar symmetry-breaking bifurcation at the same length of the recirculation bubbles $(L \approx 6)$ as ours (but at much higher $R e$ ).

The stability analysis and control of the inertia-dominated flow in an $\mathrm{X}$-junction is also interesting from a fundamental point of view because the flow contains confined jet-like structures and recirculation regions. The flow in a planar X-junction exhibits two bifurcations as the Reynolds number is increased from zero. A similar bifurcation pattern has been observed in a channel with a sudden expansion (Fearn et al. 1990; Fani et al. 2012), contraction (Chiang \& Sheu 2002), or both (Mizushima \& Shiotani 2000). The flow first bifurcates to an asymmetric steady state through a pitchfork bifurcation. Further increasing the Reynolds number, the flow develops a time-dependent nonlinear cycle, initiated by a stationary global instability of a single recirculation bubble. Similar instabilities are observed in the flow behind a bump (Gallaire et al. 2007; Passaggia et al. 2012), behind a backward facing step (Barkley et al. 2002; Marquet et al. 2009; Lanzerstorfer \& Kuhlmann 2011), in boundary layer recirculation bubbles (Rodriguez \& Theofilis 2010) and in a lid-driven cavity (Albensoeder et al. 2001; Haque et al. 2012).

Adjoint-based sensitivity analysis of flows was first introduced by Hill (1992) and has been used extensively to examine the receptivity to internal and external modifications (Sipp et al. 2010; Luchini \& Bottaro 2014). In this study, we use a global stability and a structural sensitivity analysis to investigate the dynamics of instabilities in a planar X-junction flow. Structural sensitivity analysis was introduced by Chomaz (2005) and Giannetti \& Luchini (2007) to identify the origin of instabilities in open shear flows. The structural sensitivity is defined as the region of the flow where a force-velocity feedback results in the largest drift of the eigenvalue. In this paper, we also seek the most efficient control strategy to delay or promote the first pitchfork bifurcation. We use the sensitivity to boundary velocity modification, introduced by Hill (1992) and used among others in Marquet \& Sipp (2010), to obtain an optimal suction/blowing distribution at the wall. Furthermore, we map the control effect on the flow by using the base flow sensitivity (Marquet, Sipp \& Jacquin 2008; Pralits, Brandt \& Giannetti 2010). Similar techniques were used by by Meliga and Chomaz (2011) on the flow in a confined impinging jet. They employed an adjoint based method to control the global modes by optimal body and wall forcing on a broadly comparable geometry to that in the present work. Promoting the first and second bifurcations is useful in order to increase mixing by large-scale structures, e.g. in inertial micromixers, while delaying the first bifurcation is useful in order to obtain mixing by diffusion or maintain symmetric flow, e.g. in flow focusers for fibre fabrication. We also aim to shed further light on the physical mechanisms behind the first bifurcation, and proposed control strategies.

This paper is organized as follows. We describe the flow configuration, the governing 


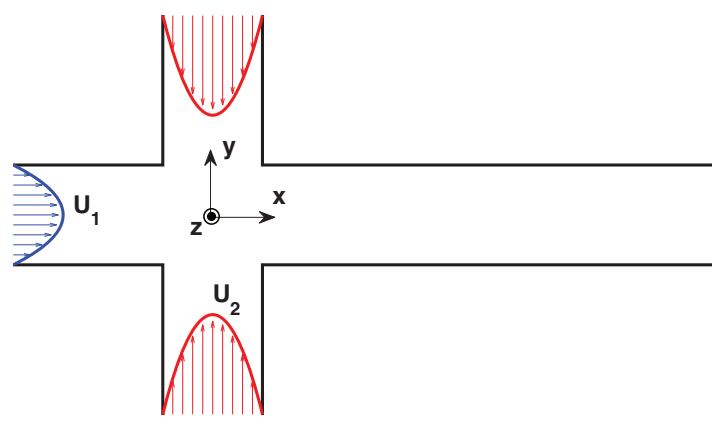

FiguRE 1. Schematic of the geometrical configuration and coordinate system

equations and the stability formulations in $\S 2$. We introduce the numerical methods and validation in $\S 3$. We report the results of the stability analysis and control of the first bifurcation in $\S 4$, and the results of the second bifurcation in $\S 5$. The conclusions are presented in $\S 6$.

\section{Configuration and problem formulation}

The flow setup and coordinate system are shown in figure 1. The geometry consists of a main channel aligned with the $x$-axis, and two identical side channels attached perpendicularly to the main channel at the same position, forming a cross. The origin of the coordinate system is located at the centre of the cross. The mean flow is homogenous in the spanwise direction $(z)$. The geometry comprises three inlets (one for the main channel and two for the side channels) and one outlet (for the main channel). The side channels have the same inflow conditions. The flow is governed by the non-dimensional incompressible Navier-Stokes and continuity equations,

$$
\begin{array}{r}
\frac{\partial \mathbf{u}}{\partial t}+\mathbf{u} \cdot \nabla \mathbf{u}=-\nabla p+\frac{1}{R e} \nabla^{2} \mathbf{u} \\
\nabla \cdot \mathbf{u}=0,
\end{array}
$$

where $\mathbf{u}=(u, v, w)$ is a vector of nondimensional velocity components, and $p$ is the non-dimensional pressure. Two non-dimensional parameters define the characteristics of the flow: the Reynolds number $(R e)$ and the velocity ratio $\left(V_{r}\right)$. The Reynolds number is defined as

$$
R e=\frac{U_{1} h}{\nu}
$$

where $U_{1}$ is the maximum inflow velocity of the main channel, $h$ is the half width of the channels and $\nu$ is the kinematic viscosity. The velocity ratio is defined as

$$
V_{r}=\frac{U_{2}}{U_{1}}
$$

where $U_{2}$ is the maximum inflow velocity of the side channels. In this paper, we study the stability of the $\mathrm{X}$-junction flow in the Reynolds number regime $60<R e<700$ and velocity ratio $0.5<V_{r}<4$. The choice of the value of $V_{r}$ is based on the experiments by Tomas et al. (2010) and Håkansson (2012). 


\subsection{Linear stability analysis}

In order to perform a linear stability analysis, we decompose the flow into a twodimensional steady base flow and an infinitesimal three-dimensional unsteady perturbation. The base flow and perturbation velocities and pressure are denoted by $Q=\left(\mathbf{U}_{b}, P_{b}\right)$ and $q=\left(\mathbf{u}^{\prime}, p^{\prime}\right)$ respectively. The perturbation $q$ takes the form of two-dimensional global modes,

$$
\begin{aligned}
\mathbf{u}^{\prime}(x, y, z, t) & =\hat{\mathbf{u}}(x, y) \exp (\sigma t+i \beta z) ; \\
p^{\prime}(x, y, z, t) & =\hat{p}(x, y) \exp (\sigma t+i \beta z),
\end{aligned}
$$

where the span-wise wavenumber, $\beta$, exploits the homogeneity of the base flow in the span-wise direction. For a given $\beta$, the complex frequency, $\sigma$, can be computed by solving the linearized eigenvalue problem,

$$
\begin{array}{r}
\sigma \hat{\mathbf{u}}+\hat{\mathbf{u}} \cdot \nabla \mathbf{U}_{b}+\mathbf{U}_{b} \cdot \nabla \hat{\mathbf{u}}=-\nabla \hat{p}+\frac{1}{R e} \nabla^{2} \hat{\mathbf{u}} \\
\nabla \cdot \hat{\mathbf{u}}=0 .
\end{array}
$$

To compute the base flow, we set up parabolic inflow conditions for all the inlets and a stress free outflow condition for the outlet. For the stability analysis, we set a Dirichlet boundary condition for all the inlets and traction free boundary condition for the outlet as suggested by Barkley et al. (2008). The total field (the base flow plus the perturbation) satisfies the outlet boundary condition. The real and imaginary part of the eigenvalue $\sigma$ are the growth rate and oscillation frequency of the corresponding global mode. If there exists any eigenvalue with $\Re\{\sigma\}>0$, the flow is linearly unstable. Conversely, if all eigenvalues have $\Re\{\sigma\}<0$, the flow is linearly stable and infinitesimal perturbations eventually decay to zero.

\subsection{Structural sensitivity}

The generalized Lagrange identity is used to derive the adjoint equations. We introduce a similar ansatz for the non-trivial solution of the adjoint system:

$$
\begin{aligned}
\mathbf{u}^{\prime+}(x, y, z, t) & =\hat{\mathbf{u}}^{+}(x, y) \exp (-\sigma t+i \beta z) ; \\
p^{\prime+}(x, y, z, t) & =\hat{p}(x, y)^{+} \exp (-\sigma t+i \beta z),
\end{aligned}
$$

where $\mathbf{u}^{\prime+}=\left(u^{\prime+}, v^{\prime+}, w^{\prime+}\right)$ and $p^{\prime+}$ are the adjoint velocity and pressure perturbation fields. The adjoint eigenvalue problem is:

$$
\begin{array}{r}
-\sigma \hat{\mathbf{u}}^{+}-\nabla \mathbf{U}_{b} \cdot \hat{\mathbf{u}}^{+}+\mathbf{U}_{b} \cdot \nabla \hat{\mathbf{u}}^{+}=-\nabla \hat{p}^{+}-\frac{1}{R e} \nabla^{2} \hat{\mathbf{u}}^{+} \\
\nabla \cdot \hat{\mathbf{u}}^{+}=0 .
\end{array}
$$

We calculate the direct and adjoint global modes to construct the structural sensitivity (Giannetti \& Luchini 2007). This gives the core of the instability, defined as the region where a local feedback force proportional to the velocity results in the largest drift of the leading eigenvalue. The sensitivity tensor is:

$$
\mathbf{S}\left(x_{0}, y_{0}\right)=\frac{\hat{\mathbf{u}}^{+}\left(x_{0}, y_{0}\right) \hat{\mathbf{u}}\left(x_{0}, y_{0}\right)}{\int_{D} \hat{\mathbf{u}}^{+} \cdot \hat{\mathbf{u}} d A} .
$$

The core of the instability can be identified by different norms of the tensor $\mathbf{S}$. Here we use the spectral norm, which measures the effect of the maximum possible coupling among the velocity components. 


\subsection{Base flow sensitivity}

The sensitivity to generic base flow modifications, $\mathbf{S}_{B F}$, where the modified base flow is not necessarily a solution to the steady Navier-Stokes equations, represents the variation of the complex eigenvalue, $\delta \sigma$, with respect to the small modification of base flow, $\delta \mathbf{U}$. The sensitivity to base flow modification is given by (see Marquet et al. 2008)

$$
\mathbf{S}_{B F}=\frac{-(\nabla \hat{\mathbf{u}})^{H} \cdot \hat{\mathbf{u}}^{*}+\nabla \hat{\mathbf{u}}^{+} \cdot \hat{\mathbf{u}}^{*}}{\int_{D} \hat{\mathbf{u}}^{+} \cdot \hat{\mathbf{u}} d A},
$$

where ${ }^{*}$ and ${ }^{H}$ stand for complex conjugate and transpose conjugate respectively. We denote the horizontal and vertical component of the sensitivity to base flow modification by $S_{U}$ and $S_{V}$. Note that for a zero-frequency mode, $\mathbf{S}_{B F}$ is a real quantity. This is because two-dimensional modifications of the two-dimensional base flow can only change the growth rate of the mode, while the frequency remains zero.

\subsection{Sensitivity to boundary velocity modification}

The sensitivity to boundary velocity modification, $\mathbf{S}_{b}$, can be derived by explicitly including the boundary condition as a constraint in the Lagrangian functional, and considering the boundary velocity $\mathbf{U}_{B}$ as a control variable. A procedure similar to Marquet et al. (2008); Marquet \& Sipp (2010), then gives:

$$
\mathbf{S}_{b}=P^{+} \mathbf{n}+R e^{-1} \nabla \mathbf{U}^{+} \cdot \mathbf{n},
$$

where $\mathbf{U}^{+}$and $P^{+}$are the adjoint base flow velocity and pressure. The eigenvalue drift is obtained by integrating $\mathbf{S}_{B}$ over the domain boundary $B$ :

$$
\delta \sigma=\int_{B} \mathbf{S}_{b} \cdot \delta \mathbf{U}_{B}
$$

\section{Numerical methods}

We compute the base flows with the spectral element method, SEM, implemented in the code nek5000 (see Tufo \& Fischer 1999). In this approach, the computational domain is divided into quadrilateral spectral elements. The equations are cast into weak form and discretized following the $\mathbb{P}_{N}-\mathbb{P}_{N-2}$ SEM discretization by Maday \& Patera (1989). The velocity space consists of $N^{t h}$-order Lagrange polynomial interpolants, $h_{i}^{N}(x)$, based on tensor-product arrays of Gauss-Lobatto-Legendre (GLL) quadrature points in a local element, $\Omega^{e}, e=1, \ldots, E$, satisfying $h_{i}^{N}\left(\xi_{j}^{N}\right)=\delta_{i j}$, where $\xi_{j}^{N} \in[-1,1]$ denotes one of the $N+1$ GLL quadrature points and $\delta_{i j}$ is the Kronecker delta. SEM combines the geometrical flexibility of finite elements with the high accuracy of spectral methods. It results in exponential convergence of the solution when the polynomial order is increased, called $p$-refinement (see Patera 1984). It can also be used for localized refinement by increasing the number of elements, called $h$-refinement.

We use the stability solver DOG (Barkley et al. 2008) to investigate three-dimensional instabilities about the two-dimensional base flows. The perturbation fields are considered as Fourier modes and dealt with in Fourier-transformed space. A time stepping technique is used to calculate the evolution of the direct and adjoint equations. A built-in library, together with ARPACK, calculates the eigen-solution of the stability problem.

The three-dimensional global modes $(\beta \neq 0)$, discussed in $\S 4.2 .4$, are computed using the FreeFem++ open-source finite element software (Pironneau et al. 2013), validated in $\S 3.1$ against Nek5000 and DOG. For this we use $P_{2}-P_{1}$ Taylor-Hood elements. 


\begin{tabular}{c|ccc}
\hline Reynolds number & coarser mesh & finer mesh & relative error $(\%)$ \\
\hline 80 & $0.0-0.0626 \mathrm{i}$ & $0.0-0.0633 \mathrm{i}$ & 0.0111 \\
90 & $0.0+0.0476 \mathrm{i}$ & $0.0+0.0472 \mathrm{i}$ & 0.0084 \\
100 & $0.0+0.1288 \mathrm{i}$ & $0.0+0.1285 \mathrm{i}$ & 0.0023
\end{tabular}

TABLE 1. Influence of the grid resolution on the growth rate of the leading eigenvalues

\begin{tabular}{c|ccc}
\hline Code & inlet length & outlet length & growth rate \\
\hline Nek5000 & 4 & 40 & 0.0476 \\
Nek5000 & 8 & 40 & 0.0811 \\
Nek5000 & 16 & 40 & 0.0815 \\
Nek5000 & 8 & 80 & 0.0811 \\
DOG & 8 & 40 & 0.0807 \\
FreeFem++ & 8 & 40 & 0.0813
\end{tabular}

TABLE 2. Influence of the domain size on the growth rate of the leading eigenvalues at $\operatorname{Re}=90$

\subsection{Validation}

In this section, we show the effect of the resolution and domain length on the stability of the flow in order to validate the numerical methods. We ensure that the leading eigenvalue does not vary when the number of elements and the polynomial order, $N_{p}$, are increased, and also when the inlets and outlet channels are lengthened. These results are summarized in tables 1 and 2. In the first study, we compare two meshes: a coarser mesh with 1072 elements and $N_{p}=8$, and a finer mesh with 2544 elements and $N_{p}=8$. The inlet and outlet lengths are 4 and 40 respectively, while the width of the main and side channels are 2 . Table 1 shows that the growth rate of the leading eigenvalue changes by less than $0.1 \%$ so we choose the coarser mesh for the rest of this study. In the second study, documented in table 2, we quantify the effect of the domain length on the stability, employing three different numerical codes. Based on this result, we choose the inlet lengths to be 8 and the outlet length to be 40 . These tests shows a very good agreement between the results obtained with the three different stability solvers.

\section{First bifurcation}

\subsection{Linear stability analysis}

For $V_{r}=3$ and Reynolds number $R e<82.5$, the DNS converges to a steady and stable symmetric flow in the X-junction (see figure 2a). At the beginning of the outlet channel, a symmetric jet forms and establishes two recirculating regions. Further downstream, the flow recovers the Poiseuille channel flow profile due to the action of viscous dissipation. As the Reynolds number increases, a steady and stable asymmetric state appears (see figure $2 \mathrm{~b}$ ). At the start of the outlet channel, the jet tilts towards one of the walls and the two recirculation regions have unequal sizes. There is an equal probability for the jet to tilt towards the upper or lower wall. The same phenomenon has been observed in similar flow configurations (see the experimental studies by Cherdron, Durst \& Whitelaw (1978) and Fearn, Mullin \& Cliffe (1990) on the laminar flow over a planar symmetric sudden expansion). We define the Reynolds number at which the flow changes its state 
(a)

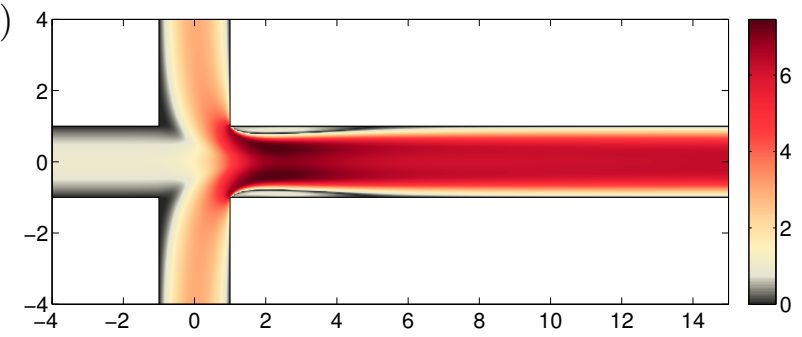

(b)

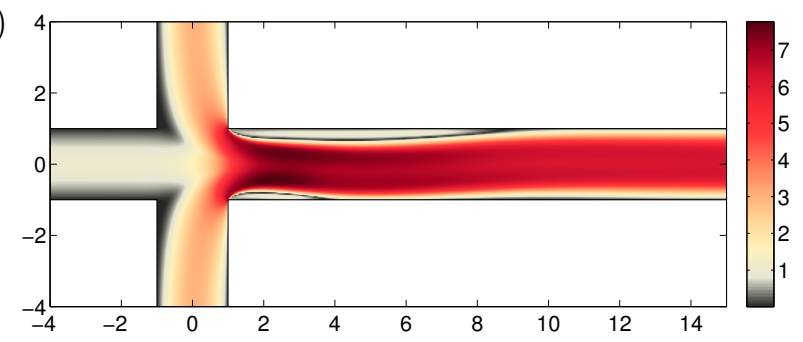

FiguRE 2. Horizontal velocity distribution for $V_{r}=3(a)$ symmetric steady state at $R e=80$

(b) asymmetric steady state at $R e=110$

from steady symmetric to steady asymmetric, as the critical Reynolds number of the first bifurcation, $R e_{c 1}$. The attachment of a jet to the adjacent wall, the Coanda effect, has been studied for some time (see the early work by Bourque \& Newman 1960). It is understood as a consequence of the pressure difference established in the cross-stream direction (see e.g Sobey \& Drazin 1986). To better understand the nature of the first bifurcation, we perform a global stability analysis around the symmetric base flow. In order to obtain the steady but unstable symmetric base flow at $R e>R e_{c 1}$, we simulate the flow in only half of the domain, imposing the symmetry boundary condition on the axis of symmetry. The global analysis in the full domain around this symmetric flow at $R e>R e_{c 1}$ has stationary unstable modes for all velocity ratios. In the linear framework, the flow therefore becomes globally unstable through a pitchfork bifurcation. In figure 3(a), we show the growth rate of the leading global modes as a function of the span-wise wavenumber, $\beta$, for the symmetric basic flows at $R e=82.5,90$ and 115 and for $V_{r}=3$. The most unstable mode has zero span-wise wavenumber; i.e the first bifurcation is two-dimensional, and the critical Reynolds number for the first bifurcation is 82.5 . The eigenvalue spectrum of the flow at $R e=82.5$ is shown in figure $3(\mathrm{~b})$ for a zero span-wise wavenumber. The distribution of the eigenvalues is symmetric around the zero frequency axis, and the least stable eigenvalues are discrete (well separated from the continuous spectrum further down in the figure).

Figure 4 shows the spatial structure of the most unstable global mode for $R e=90$ and $\beta=0$. Only part of the domain is shown; the domain size is larger in the simulations. The global modes have highest amplitude around the recirculation bubble and the $u$ perturbation is the strongest. This increases the speed at the outer edge of the bottom bubble and decreases the speed at the outer edge of the top bubble. The $v$-perturbation moves the flow down around $x=2$ and up around $x=6$. The $p$-perturbation shows that the pressure decreases in the bottom bubble around $x=4$ and increases in the top bubble. The structure of the global modes suggest that the streamwise acceleration leads to lower pressure at the lower recirculation zone (the opposite for the top bubble), which in turn induces a cross-stream pressure gradient which helps to maintain the asymmetry. 
(a)

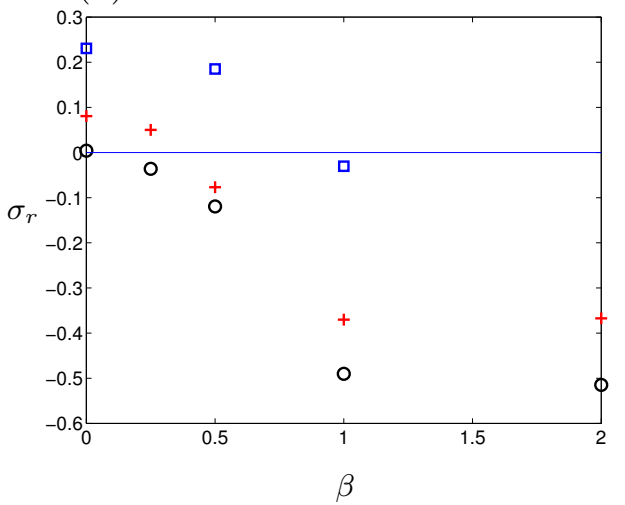

(b)

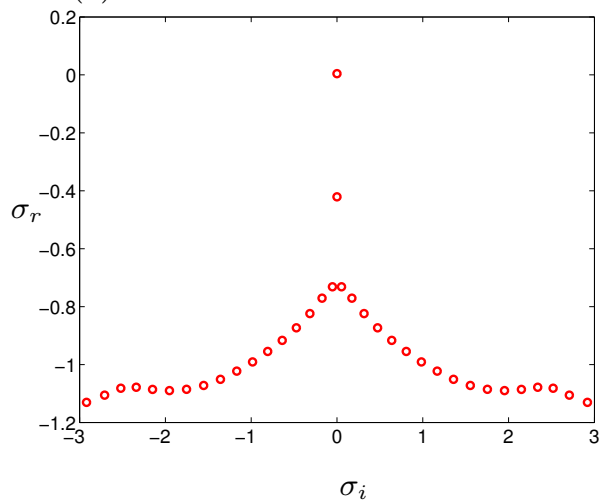

FigURE 3. (a) Growth rate of the leading eigenvalues about the symmetric base flows for $V_{r}=3$ and $\operatorname{Re}=82.5($ black $\bigcirc)$, 90(red + ) and 115(blue $\square)(b)$ Eigenvalue spectrum for $V_{r}=3$, $R e=82.5$ and $\beta=0$

(a)

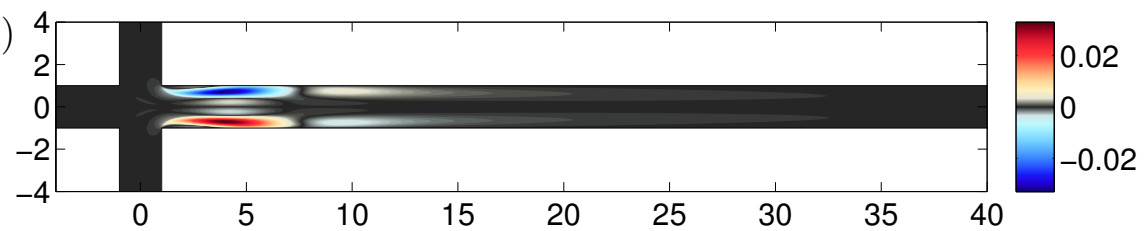

(b)

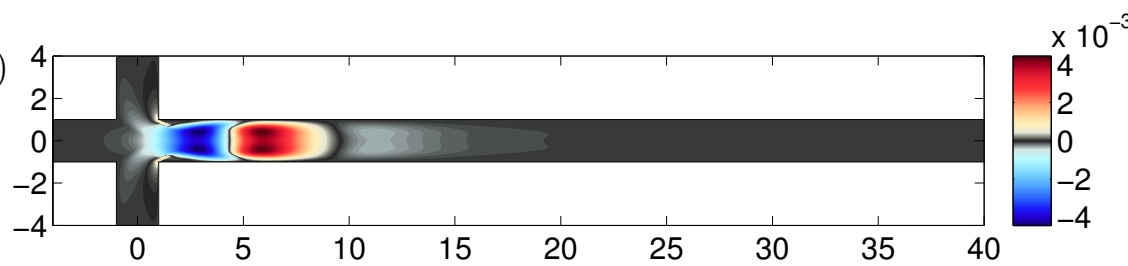

(c)

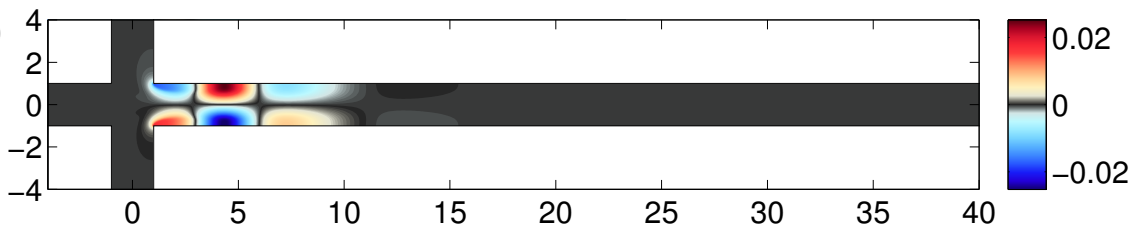

Figure 4. Spatial structure of the direct global modes, $(a)$ horizontal velocity, $(b)$ vertical velocity, and $(c)$ pressure around the symmetric base flow at $R e=90, V_{r}=3$ and $\beta=0$

This effect is similar to the asymmetric attachment in stenotic flow which is described in the work by Sherwin \& Blackburn (2005): "the axial perturbation velocities induce a weak cross-flow pressure gradient and flow... Therefore the perturbation flow tends to deflect the centreline of the enclosed jet away from the tubes axis of symmetry, promoting a mild Coanda-type attachment". The growth of the $u$-perturbation itself can be explained by a lift-up mechanism, as will be detailed in a later paragraph.

The region with the highest structural sensitivity, which is also known as the core of the instability, is the region of the flow in which force-velocity feedback has the greatest influence on the eigenvalue. This core is found by overlapping the direct and adjoint 
global modes and is shown in figure 5(a). It is localized at the edge of the recirculation bubble(s), suggesting that the instability can be controlled by manipulating the bubble. This will be confirmed in $\S 4.2$, where passive control by optimal suction/blowing at the wall is examined. Here we depict only the spectral norm of the sensitivity tensor. The $u^{+} u$ component of the sensitivity tensor is significantly stronger than the other components and has the same structure as the spectral norm.

In figure 5(b), we present the spatial map of the production of the perturbation kinetic energy for the same flow. The production term is normalized by the overall perturbation kinetic energy in the domain. The regions of largest transfer of the kinetic energy from the base state to the perturbations are found in the shear layer at the edge of recirculation bubbles. Interestingly, the core of the instability corresponds to the region of the maximum positive and negative production.

More specifically, we compute the contribution of the term $u v d U / d y$ in the production of the perturbation kinetic energy. The spatial map of this term is depicted in figure $5(\mathrm{c})$, and can be compared to the map of total energy production in $5(\mathrm{~b})$. We observe that total energy production and energy production by the term $u v d U / d y$ are almost identical in amplitude and shape, from which we conclude that the instability growth is due to this term.

The disturbance extracts energy from the base flow through the $u v d U / d y$-term in several shear flow instabilities, most notably, the Kelvin-Helmholtz instability, TollmienSchlichting waves, and the lift-up effect. To determine the instability mechanism in our flow, we note that the present instability is stationary, the mode shape streamwise elongated, and the streamwise velocity component order of magnitude larger than the vertical component (figure $4 \mathrm{a}$ and $\mathrm{b}$ ). This strongly indicates a lift-up mechanism, where a small initial $v$-perturbation induces a strong $u$-perturbation (for a review on lift-up see Brandt (2014)).

This instability is different from the lift-up in parallel flows due to the feedback provided by the recirculation region, which leads to a self sustained mechanism. This is similar to the finding of Kuhlmann 2011 who note that the instability behind a backwardsfacing step is caused by a lift-up effect, which gains its exponential growth through recirculation for some specific value of the step height. The present configuration with two symmetric bubbles is more unstable than the case of a single bubble, because the 2D asymmetric instability mode sets in at lower Reynolds numbers. This can also be explained by the energy budget: the antisymmetric global mode involving two bubbles allows the $v$-perturbation to be constant in the vertical direction, minimizing $d v / d y$, and thereby minimizing the global dissipation.

The critical Reynolds number for the first bifurcation depends significantly on $V_{r}$. Figure 6(a) shows that the critical Reynolds number decreases monotonically from about 530 for $V_{r}=0.5$ to about 60 for $V_{r}=4$. Nonlinear simulations on the two-dimensional domain give the same result for the first bifurcation (not shown here). If we define the Reynolds number based on the maximum velocity at the side channels, however, the neutral curve for the first bifurcation is almost independent of the velocity ratio: $R e_{c}=262.5-245$ for $V_{r}=0.5-4$. We also examine two extreme cases: $V_{r}=10$ and $V_{r}=0.2$. For $V_{r}=10, R e_{c} \approx 260$ based on $U_{2}$, similarly to the previous cases. For $V_{r}=0.2$ however, $R e_{c} \approx 310$, i.e. the flow is more stable when the side flow is weaker. This shows that the critical Reynolds number of the first bifurcation is mainly determined by the inlet velocity of the side channels.

To visualize the bifurcation of the flow in the $\mathrm{X}$-junction quantitatively, we introduce a measure of the deviation from the symmetric flow: the $v$-velocity at $(x, y)=(3,0)$, on the centreline. In figure $6(\mathrm{~b})$, we show the maximum and minimum value (in time) of 

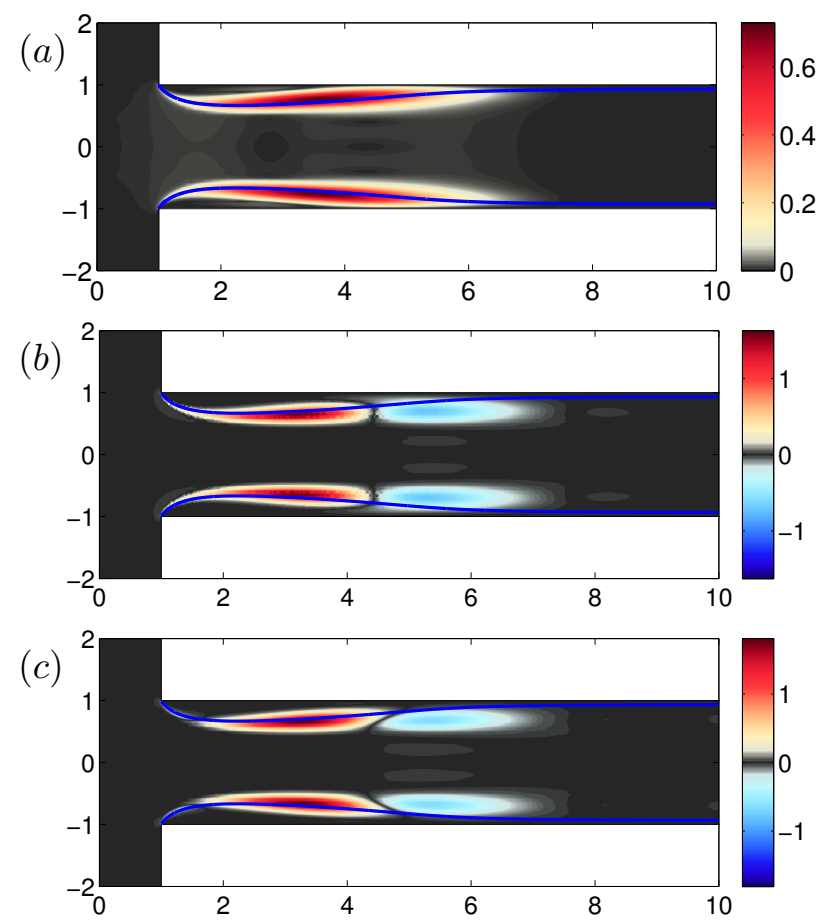

Figure 5. (a) Spatial map of structural sensitivity; (b) production of the perturbation kinetic energy; (c) lift-up perturbation kinetic energy around the symmetric base flow at $R e=90$, $V_{r}=3$ and $\beta=0$.

this measure as a function of the Reynolds number. Up to $R e=110$, the flow is steady and $v$ is constant. The first bifurcation is evident at $R e=82.5$. We will return to this figure when examining the second bifurcation.

For this flow, the point of first bifurcation seems to be determined by the size of the recirculation region. Figure 7 shows the stagnation streamline that starts from the upper right corner, $(x, y)=(1,1)$, and separates the recirculating bubble from the flow that passes through the $\mathrm{X}$-junction. For a fixed velocity ratio $V_{r}=3$, figure $7(\mathrm{a})$, the bubble elongates as the Reynolds number increases. For the cases along the neutral curve, figure $7(\mathrm{~b})$, the bubble has almost the same size for various velocity ratios and Reynolds numbers. It seems therefore that the first bifurcation occurs when the bubble reaches a critical length, which is about 6.1 .

This result also provides a physical explanation for the scaling found in figure $6(\mathrm{a})$. The shape of the recirculating region at the critical conditions is unaffected by the main stream when $V_{r}>0.5$. This is because the side flows force the horizontal stream to flow down the middle of the exit channel without affecting the dynamics of the bubbles. This explains the dependency of the bifurcation thresholds on the velocity of the side channels. For low velocity ratios however, i.e $V_{r}=0.2$, the stability boundary is different and $R e_{c}$ is higher. Based on these principles, we anticipate that the critical condition for the T-junction will be very similar to that of the $\mathrm{X}$-junction.

To investigate whether the bifurcation is supercritical or subcritical, we have performed nonlinear simulations with various amplitudes of initial noise and have observed that the final steady symmetric solution is attained for all cases when $R e<R e_{c 1}$. This is also 

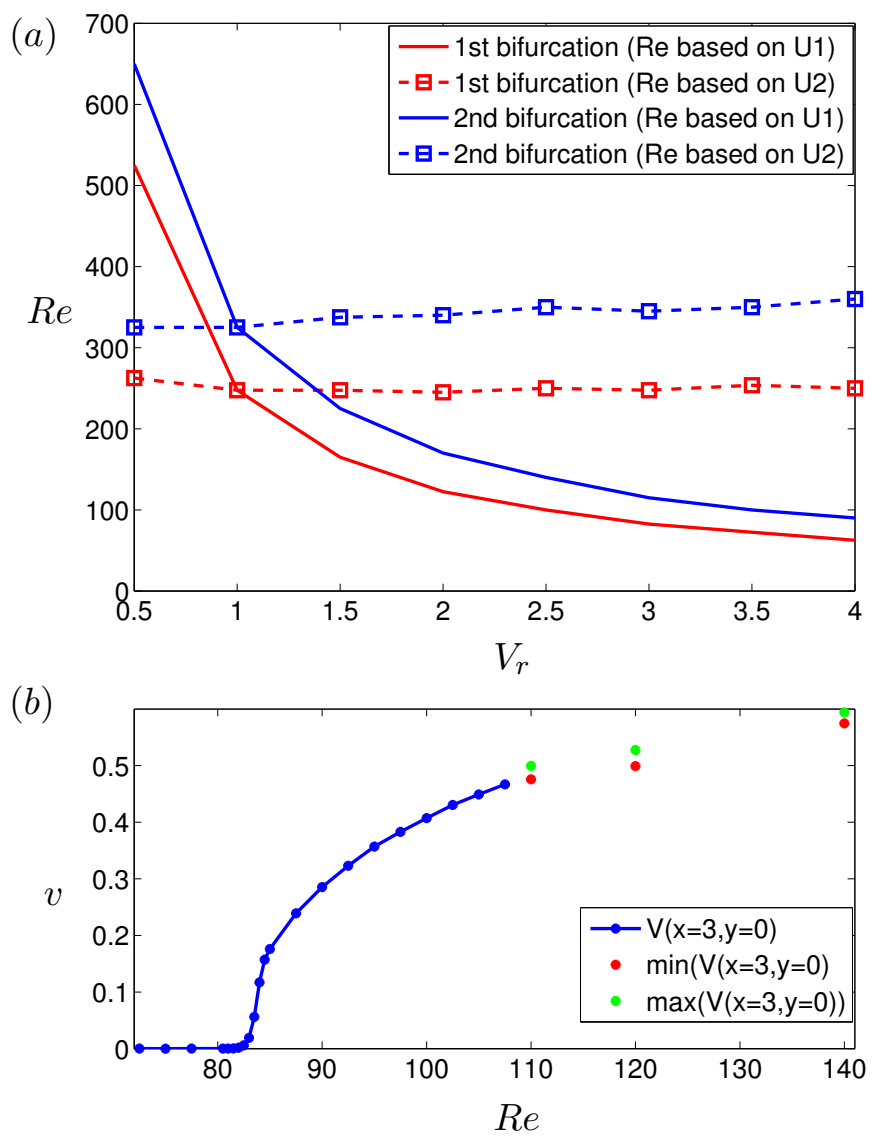

FiguRE 6. (a) Critical Reynolds number for the first and second bifurcations versus velocity ratio (b) Bifurcation diagram of the $\mathrm{X}$-junction flow with velocity ratio $V_{r}=3$

true when we simulate the flow using an asymmetric solution as the initial condition. We conclude therefore that the first bifurcation is supercritical.

\subsection{Control of the first bifurcation}

A traditional way to control globally unstable open shear flows is by a control cylinder. However, for a complex wall-bounded geometry, small amplitude suction or blowing at the walls presents a more versatile option because the amplitude distribution can be varied more easily. We therefore try to control the first bifurcation of the X-junction by small-amplitude steady (time-independent) suction or blowing at the walls.

\subsubsection{Sensitivity to wall suction}

The sensitivity $\mathbf{S}_{b}(x, y)$ represents the change in the growth rate due to a localized velocity change of amplitude $A=1$ at the boundary location $(x, y)$, in the linear limit. The eigenvalue drift for different amplitudes and finite actuation areas can be obtained from $\mathbf{S}_{b}$ by integration (Equation 2.10). Figure 8 (a) shows the real part of $S_{b, n}$, which is the change in the growth rate $\sigma_{r}$ due to control in the horizontal channel. By suction we mean a velocity at the wall in the direction of the outgoing normal. We show the sensitivity for two different Reynolds numbers: $R e=90$ (just above bifurcation) and $R e=150$ (far from bifurcation). The main observation is that the sensitivity to suction 
(a)
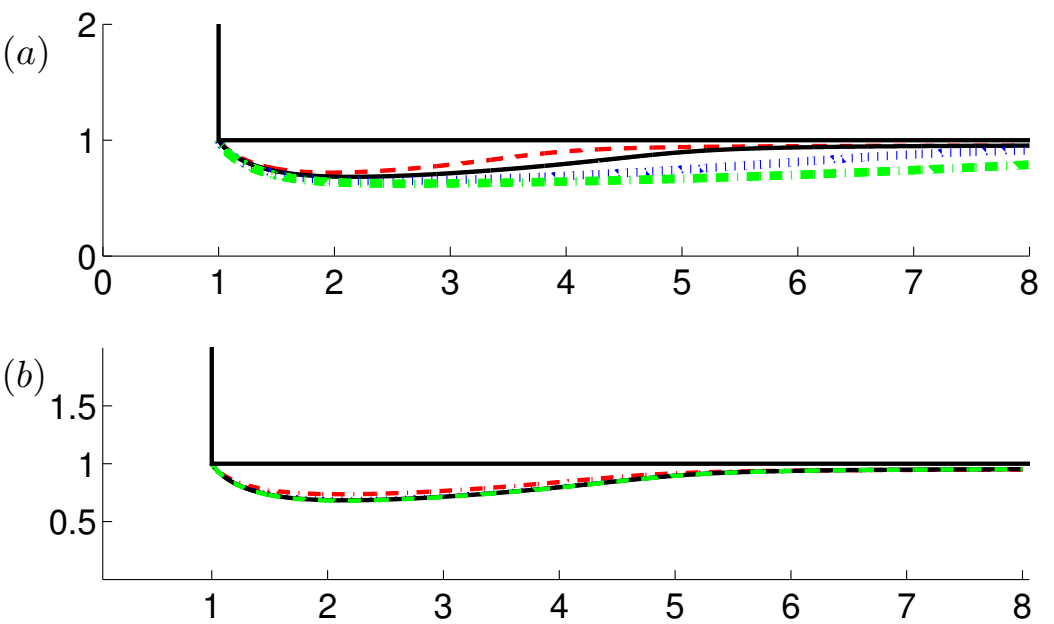

FIGURE 7 . Recirculation bubble of symmetric base flows for $(a)$ a fixed velocity ratio, $V_{r}=3$, and $R e=60$ (red line - - ), $R e=80$ (black line - ), $R e=115$ (blue line . . ) ) and $R e=162.5$ (green line - . - - .) (b) some cases along the neutral curve for $R e=520, V_{r}=0.5$ (red line - -), $R e=162.5, V_{r}=1.5$ (blue line $\left.\ldots\right), R e=80, V_{r}=3($ black line - $)$ and $R e=60, V_{r}=4($ green line - . - . -)

is largest just downstream of the junction, around $x=3$, and that suction there increases the instability growth rate, for both $R e=90$ and $R e=150$. Therefore, suction in this location is destabilizing.

The sensitivity is lower at $R e=150$ than at $R e=90$, and both stabilizing and destabilizing effects are observed for the same actuation. This indicates that a lower amplitude is needed to control the flow at $R e=90$ than at $R e=150$. Also, at $R e=150$ the departure from the steady symmetric solution will be greater than it is for the $\mathrm{Re}=90$ flow, suggesting that the sensitivity at $R e=150$ might be less relevant for control of the bifurcated flow.

Figure 8(b) shows the sensitivity along the upstream and downstream walls of the vertical channel at $R e=90$ and $R e=150$. The sensitivity is generally lower in the vertical channel than in the horizontal channel, apart from at the corners, where they are equal. The sensitivity is large and negative close to the downstream corner, which means that suction there is stabilizing. However, this region where suction is stabilizing is very small, making control near the corner difficult. Comparing the results at the same height $y$ in the upstream and downstream channel, it is interesting to note that the sensitivity is higher on the upstream wall, except at the corner.

\subsubsection{Passive control by distributed suction and blowing}

The sensitivity distributions, such as the curves in figure 8 , are also gradient distributions. This means that $\operatorname{real}\left(S_{b, n}\right)$ gives the suction distribution that increases the eigenvalue growth rate most, of any suction distribution with a given $L^{2}$-norm (and with a low enough amplitude so that the linear analysis is valid). Correspondingly, $-\operatorname{real}\left(S_{b, n}\right)$ gives the distribution that decreases the eigenvalue growth rate most. If $-\operatorname{real}\left(S_{b, n}\right)$ is applied at the boundary, the critical Reynolds number should increase.

We therefore test how far the linearly optimal distribution can increase the critical Reynolds number. The procedure is as follows. We choose a suction amplitude $A$ and first compute the base flow and modes at bifurcation; then extract the optimal stabilizing 
(a)

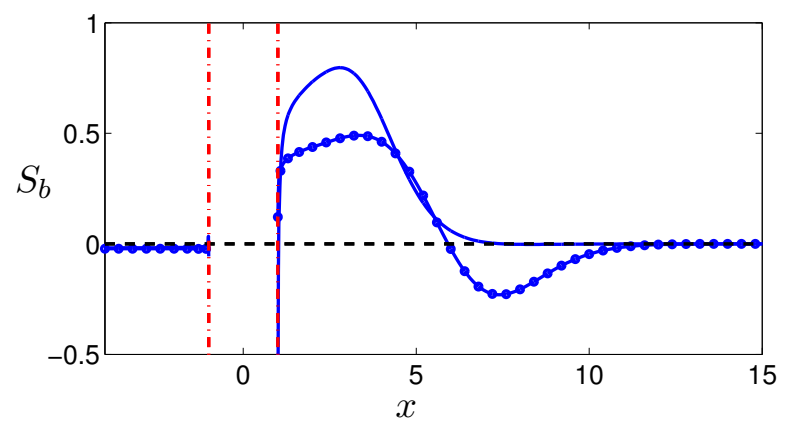

(b)

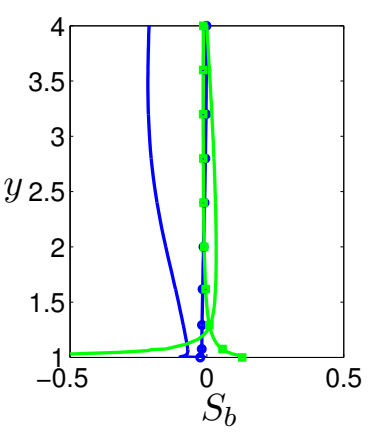

FiguRE 8. (Colour online) Sensitivity of the growth rate to suction at the main channel walls: (a) horizontal channel (w.r.t. $x$ ) and (b) vertical channel (w.r.t. $y$ ), left wall (blue) and right wall (green). $R e=90$ (plain solid line) and $R e=150$ (solid line with circles). Sensitivity to blowing is the same curve negated. The sensitivities to suction at both side channels are equal and are the same at the upper and lower walls of the horizontal channel. The minimum value of the solid line in the downstream corner is outside the axis in both subfigures: it is $\operatorname{real}\left(S_{b, n}\right)=-1.8$.

suction distribution $-\operatorname{real}\left(S_{b, n}\right)$ and scale it so that the ratio of the absolute value of the maximal suction velocity, and the maximal inflow velocity, is equal to $A$. Next, we apply the optimal suction distribution at the boundary, and recompute the base flow and the global modes. If all global modes now have negative growth rates, then all steps are repeated at higher $R e$. We keep increasing the Reynolds number until the growth rate of the leading mode is positive despite the control. In this way, we have found the new critical Reynolds number corresponding to the suction amplitude $A$.

The critical Reynolds numbers for $A=0,0.01,0.02$ and 0.03 are shown by vertical lines in figure 9 . First, it is remarkable that suction of such a small maximal amplitude as $3 \%$ of the inflow velocity can stabilize the flow until $R e=150$, which is almost double the critical Reynolds number of the uncontrolled case. Higher suction amplitudes were also tested but did not result in any further increase of the critical Reynolds number. The change of the flow rate due to suction or blowing in the configuration with $A=0.03$ is also around $3 \%$, which is small. Changes in the total flow rate due to control could be adjusted for by applying suction/blowing in the region downstream $(x>10)$, if desired. In this region, the instability is not sensitive to suction or blowing, and hence the flow rate adjustment would not interfere with the control effect.

The linear growth rate without suction is also shown in the same figure and reaches values up to $\sigma_{r}=0.27$ at $R e=150$. The growth rate with the linearly optimal suction still becomes negative at this $R e$ (the flow is stabilized). Therefore suction/blowing is a robust and efficient way to control the $\mathrm{X}$-junction flow. Next, we will investigate how the control works in more detail.

\subsubsection{Physical interpretation of suction control}

The optimal suction distribution has several simultaneous effects on the base flow. We cannot create arbitrary base flow changes because the base flow has to satisfy the Navier-Stokes equations. Some of the changes induced by the optimal suction might have a large effect on the growth rate, and some might have no effect at all but be merely a consequence of the fact that the base flow has to satisfy the Navier-Stokes equations. To find out where the effective base flow changes induced by suction are located, we investigate the product of the base flow sensitivity (Eq. 2.8) and the base flow change: $\int_{B} \mathbf{S}_{B F} \cdot\left(\mathbf{U}_{s}-\mathbf{U}_{0}\right)$, where $\mathbf{U}_{s}$ is the base flow velocity vector with suction, and $\mathbf{U}_{0}$ the 


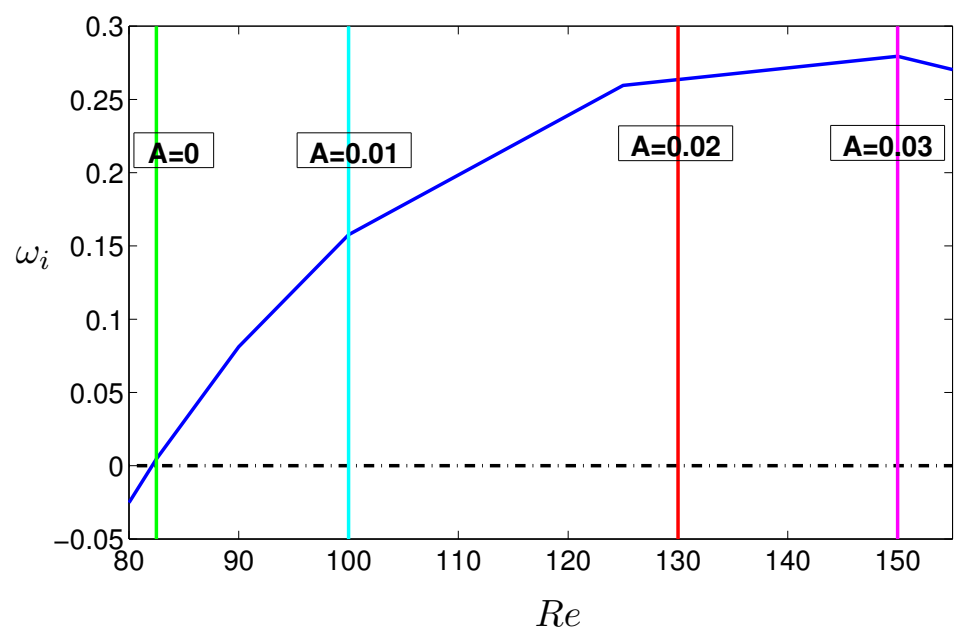

Figure 9. (Colour online) The effect of optimal boundary suction/blowing. The curve (blue online) gives the global mode growth rate of the uncontrolled base flow. The vertical lines (colour online, labeled) give the stability limits at different values of suction.

base flow velocity vector without suction. This product gives a spatial map of the control effect, and the real part, displaying stabilization, is shown in figure 10 for $R e=90$ and $R e=150$. Inside and just outside the recirculating region, the suction induces stabilizing and destabilizing base flow modifications respectively. It can be seen that the stabilizing influence occurs almost entirely inside the recirculation bubble, where the wavemaker defined by the structural sensitivity (figure 5 ) is located. This is true for both $R e=90$ and $R e=150$, although the optimal suction distributions themselves (figure 8) are qualitatively different. Hence, in line with the stability results, we find the suction to be optimal because of its effect on the most influential region of the flow: the recirculation bubble.

To quantify in more detail how the control changes the recirculation bubble, we investigate three base flows at $R e=90$ : (a) without suction/blowing, (b) with optimal stabilizing suction/blowing $-\operatorname{real}\left(S_{b, n}\right)$, and (c) with optimal destabilizing suction/blowing $\operatorname{real}\left(S_{b, n}\right)$ (see figure 11). The most stable flow has the fewest streamlines with reverse flow and the smallest recirculation bubble - i.e. there is both weaker and less recirculation. By looking at $-\operatorname{real}\left(S_{b, n}\right)$ (the negative of the curve in figure 8a), we observe that this is achieved by blowing from the wall in the location of the recirculation bubble. The attenuation of the instability can be attributed to two factors. Firstly, blowing decreases the velocity gradient between the main stream and the bubble, decreasing $v d U / d y$ and hence weakening the energy production by lift-up. Secondly, blowing also counteracts the recirculation, which is needed to produce exponential growth of the otherwise algebraic lift-up mechanism.

\subsubsection{Control of three-dimensional modes}

Previously, we showed that the optimal wall suction distribution can be computed for the leading two-dimensional eigenmode, and stabilizes that mode up to $R e=150$. However, to confirm that the flow is indeed stabilized by suction, we also need to stabilize all three-dimensional eigenmodes, $\beta \neq 0$. It is impossible to experimentally design different controls for different wavenumbers. Therefore we proceed to examine whether 

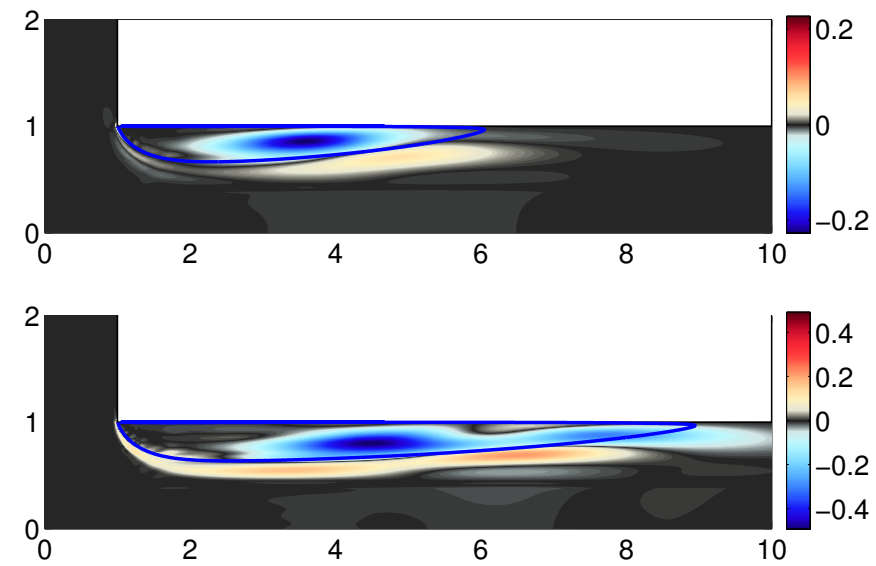

Figure 10. (Colour online) Spatial map of the control effect obtained by wall suction, $\int_{B} \operatorname{real}\left(\mathbf{S}_{B F}\right) \cdot\left(\mathbf{U}_{s}-\mathbf{U}_{0}\right)$, for (a) $R e=90$ and (b) $R e=150$. Thin blue line show the boundary of the recirculation bubble in each case.

(a)

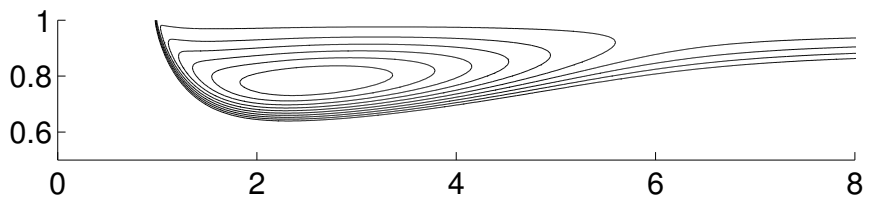

(b)

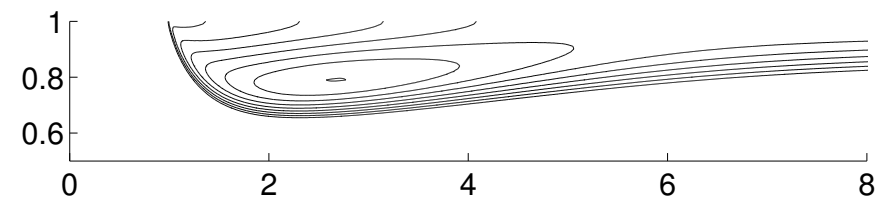

(c)

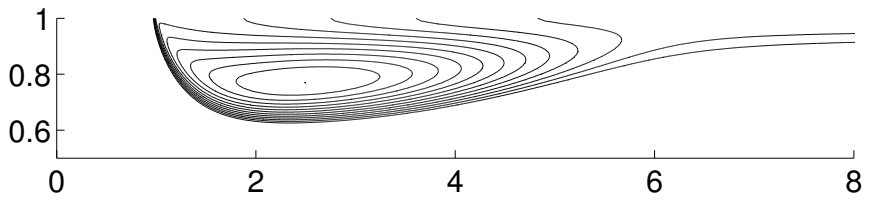

FiguRE 11. Streamlines around the recirculation bubble of the base flow: (a) Base flow with zero wall velocity, (b) Base flow with optimal stabilizing suction/blowing distribution at the wall, magnitude $1 \%$, (c) Base flow with optimal destabilizing suction/blowing distribution at the wall, magnitude $1 \%$.

the two-dimensionally optimal suction stabilizes all modes, whether optimal or not, for $\beta \neq 0$.

The leading eigenvalues for different values of $\beta$ are shown in figure 12 for the case $R e=150$, with suction and without suction. Interestingly for the cases without suction, spanwise periodic modes become gradually less and less stable by increasing the Reynolds number. The spanwise wavenumber of the least stable mode becomes different from zero at $R e \approx 120$, and approaches $\beta=0.5$ when $R e=150$. To understand this effect, we analyze the perturbation energy budget for the cases $R e=90$ and $R e=150$ at 


\begin{tabular}{ccccc}
\hline $\operatorname{Re}$ & $\beta$ & production & dissipation \\
\cline { 2 - 4 } 90 & 0 & 0.474 & -0.325 \\
90 & 0.5 & 0.296 & -0.377 \\
150 & 0 & 0.536 & -0.257 \\
150 & 0.5 & 0.599 & -0.254 \\
\hline
\end{tabular}

TABLE 3. Perturbation kinetic energy budget

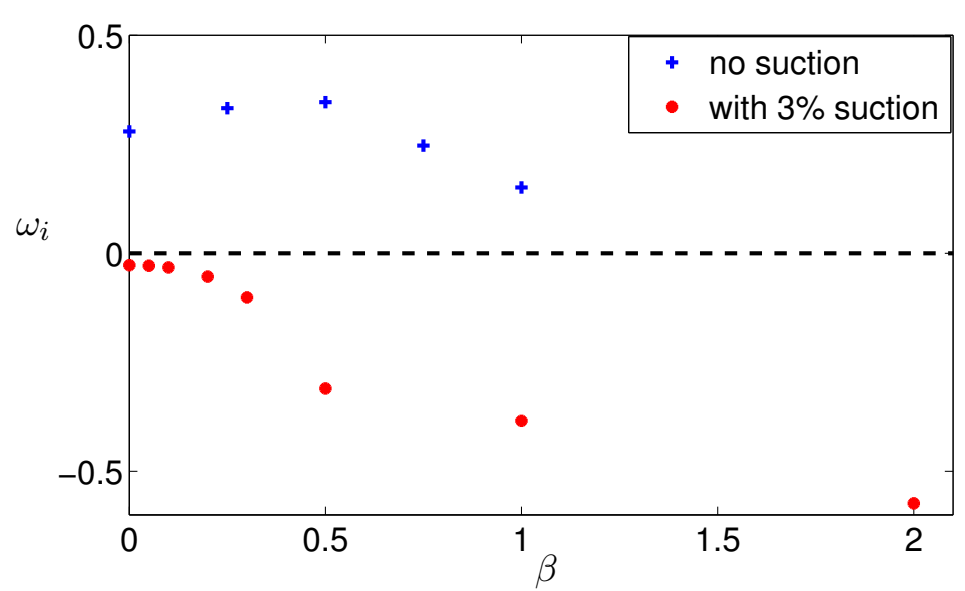

Figure 12. (Colour online) $R e=150$ : Growth rate of the most unstable eigenvalue as a function of $\beta$, with (red stars) and without (blue stars) $2 \mathrm{D}$ optimal suction

$\beta=0$ and $\beta=0.5$. The production and dissipation of the perturbation kinetic energy, normalized by the kinetic energy, are shown in table 3 . The wavenumber selection only depends on which wavenumbers experience most energy production by the lift-up effect. Dissipation does not vary significantly with $\beta$, whereas the production grows with $R e$ for $\beta=0.5$. Thus lift up is more effective at finite $\beta$ despite the presence of a spanwise velocity component in the dissipation term.

When applying control by optimal suction, we see that the eigenvalues become stable for all $\beta$. Results for Reynolds numbers lower than 150 also show the same behaviour. This confirms that the control works well for two- and three-dimensional modes, and the flow is indeed stabilized up to $R e=150$. Furthermore, we observe that the modes at nonzero spanwise wavenumbers are more affected by suction than the two-dimensional mode and the controlled flow is characterized again by a least stable mode of $\beta=0$.

\section{Second bifurcation}

\subsection{Linear analysis}

When the Reynolds number is increased beyond the first bifurcation, the flow maintains its steady asymmetric state until $R e=R e_{c 2}$, the critical Reynolds number for the second bifurcation. For $R e>R e_{c 2}$, it bifurcates again due to the action of stationary global modes around the steady asymmetric base flow. For $V_{r}=3$, a global stability analysis gives an unstable global mode with $\beta=1$ for $R e=115$. In figure 13(a), we show the 
(a)

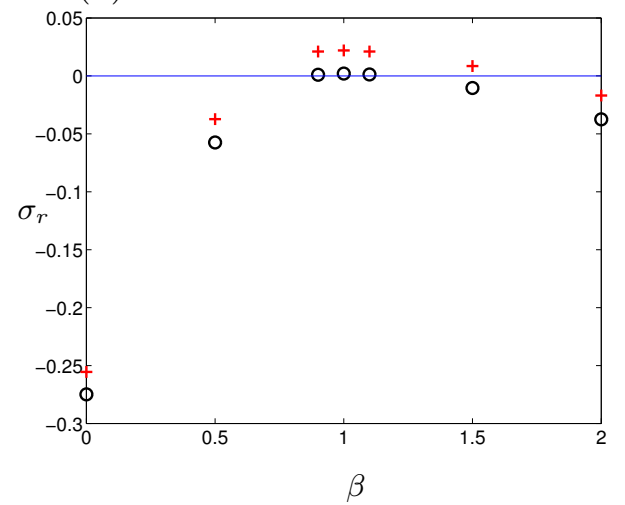

(b)

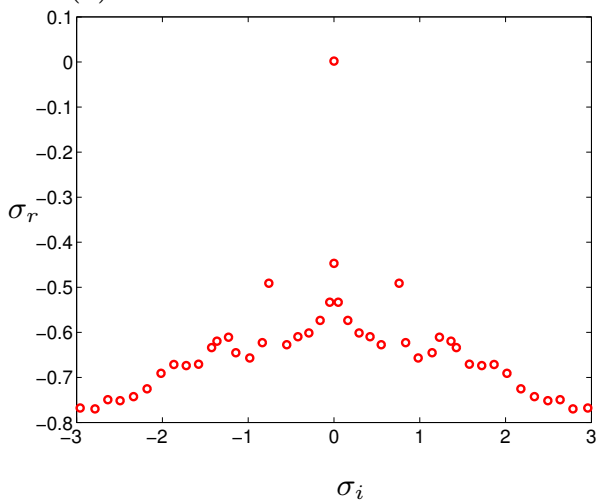

FIGURE 13. (a) Growth rate of the leading eigenvalues around the asymmetric base flows for $V_{r}=3$ at $\operatorname{Re}=115($ black $\bigcirc)$ and $120($ red +$)(b)$ Eigenvalue spectrum at $R e=115, V_{r}=3$ and $\beta=1$

leading eigenvalues as a function of the span-wise wavenumber at $R e=115$ and 120 . The second bifurcation is also of the pitchfork type. Figure 13(b) shows the eigenvalue spectrum of the asymmetric base flow at $R e=115$. The spectrum is discrete and symmetric, as for the symmetric base flows.

We present the features of the most unstable global mode around the asymmetric base flow in figure 14. The structure of the modes illustrates the asymmetry of the underlying base flow; in this case, the jet is tilted towards the lower wall. The $u$-component is the strongest and is maximal at the larger recirculation bubble, close to the upper wall of the outlet channel. The $v$ and $w$-perturbation modes also display their maximum amplitude next to the larger bubble. The strong $u$-perturbation of the global direct mode together with the strong $v^{+}$-perturbation of the global adjoint mode (not shown here), suggests that the lift up mechanism is active in the shear layer associated with the largest recirculating region (see Marquet et al. 2009). The perturbation transports the base flow momentum from the regions of low-speed to those with higher-speeds and creates a large $u$-perturbation.

Figure 15 shows the overlap of the direct and adjoint global modes, which is the core of the instability at the second bifurcation. The core is located in the larger recirculation bubble. Note that the core of the instability at the second bifurcation is in the middle of the bubble and extends over the whole bubble, while the core of the instability at the first bifurcation was localized at the downstream edges of the two bubbles (see §5.1). The instability is three-dimensional and steady, which is similar to the instabilities that develop along the streamlines in the flow behind a bump or in a cavity (see e.g Gallaire et al. 2007; Albensoeder et al. 2001; Rodriguez \& Theofilis 2010) and at the recirculation region over a backward-facing step (see e.g Marquet et al. 2009; Lanzerstorfer \& Kuhlmann 2011). Lanzerstorfer \& Kuhlmann (2011) used the energy production, together with the flow streamlines, to distinguish between three types of instability for the backwards-facing step: centrifugal, elliptic and lift-up mechanism. We shall attempt to do the same for the second bifurcation. The energy production for the instability leading to the second bifurcation is shown in figure 15 (b). The maximum of the production is in an area of curved streamlines, but the velocity increases radially outwards (to the middle stream), which precludes centrifugal instability. Furthermore, elliptic instability would be indicated by a maximum production in the centre of a vortex, while in our case the maximum is at the outer edge of the recirculation region. Similarly to the analysis of 
(a)

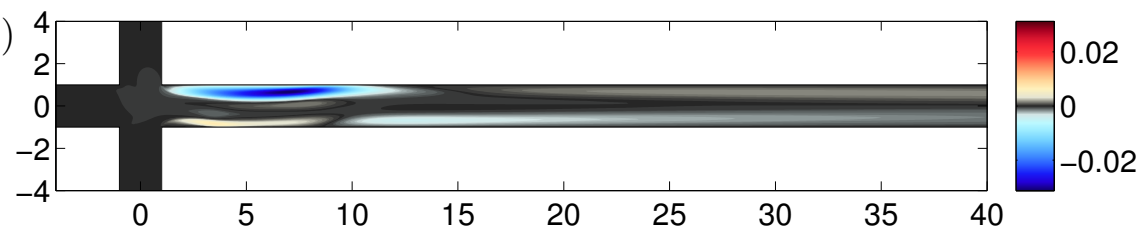

(b)

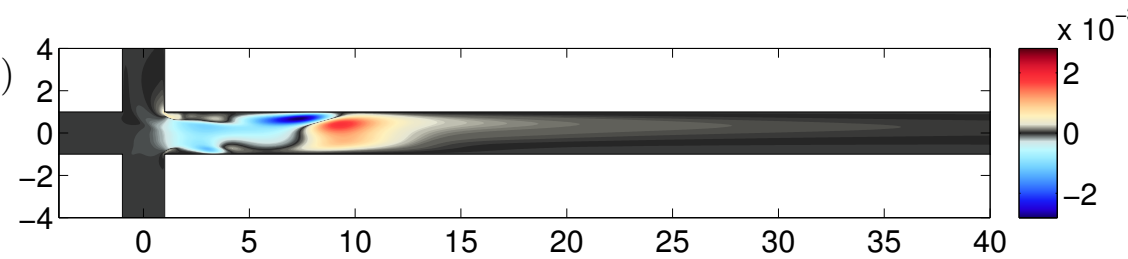

(c)

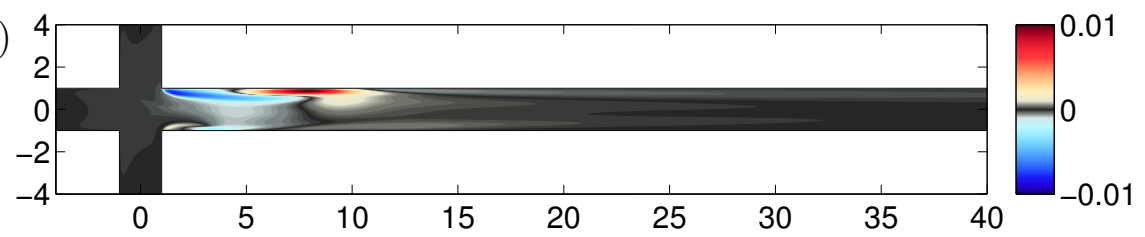

FiguRE 14. Spatial structure of the direct global modes, $(a)$ stream-wise, $(b)$ cross-stream and (c) span-wise around the asymmetric base flow at $R e=120, V_{r}=3$ and $\beta=1$

the first bifurcation, we present the spatial structure of the lift-up term $(u v D U / D y)$ in the production of the perturbation kinetic energy in 15 (c). The lift-up term presents a structure relatively similar to the total production, both with a localized maximum at the downstream edge of the larger recirculation bubble where the base flow shear is strongest. We conclude that also the second bifurcation is due to the lift-up mechanism (Lanzerstorfer \& Kuhlmann 2011), and the vertical velocity perturbation extracts energy from the base flow shear $(d U / d y)$ to produce in this case streamwise-elongated low and high speed streaks. We also show that the structural sensitivity (figure 15 a) is the largest in the middle of the bubble, indicating that velocity perturbations initiated there participate in the streamline displacement and the lift-up.

In figure 6(a), we show the critical Reynolds number for the second bifurcation. This neutral curve has the same behaviour as that of the first bifurcation. The second bifurcation is also almost independent of the velocity ratio if the Reynolds number is based on the maximum velocity in the side channel. For all velocity ratios greater than one, the most dangerous mode is found to have span-wise wavenumber $\beta=1$. However, for $V_{r}=0.5$, the mode with $\beta=1.4$ is the most unstable.

\subsection{Nonlinear analysis}

In order to further understand the results of the linear analysis about the second bifurcation, we perform nonlinear simulations on a three dimensional periodic geometry with span-wise length, $L_{z}=2 \pi$, corresponding to $\beta=1$. We use 3,6 and 9 spectral elements in the span-wise direction (the polynomial order is 8 as in the $2 \mathrm{D}$ simulations) to ensure that the final results are mesh independent. We construct an initial condition for the DNS consisting of the asymmetric base solution combined with the spanwise periodic perturbation leading to the strongest transient energy growth (computed separately by standard direct-adjoint iterations). The initial condition is scaled to have maximum span-wise velocity, the strongest component, equal to one. The non-linear evolution of the initial condition shows that the span-wise velocity decays to zero and a two-dimensional 

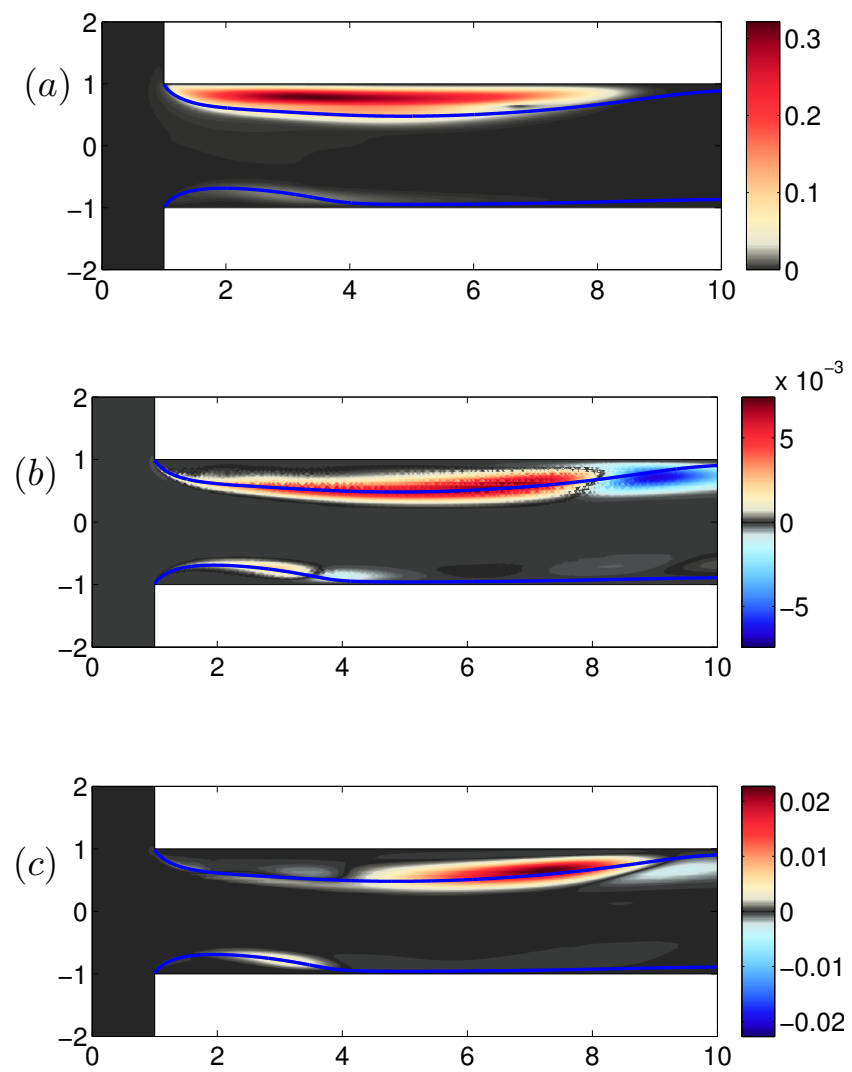

Figure 15. (a) Spatial map of the structural sensitivity, (b) Production of the perturbation kinetic energy around the asymmetric base flow at $R e=120, V_{r}=3$ and $\beta=1$, the dashed lines represent the base flow streamlines.

steady state is obtained asymptotically for all $R e \leqslant 105$ (below the second bifurcation). Interestingly, once all the disturbances have traveled out of the computational domain, the larger recirculation bubble may have moved to the opposite side with respect to the initial condition. We therefore find two possible steady two-dimensional solutions for $R e \leqslant 105$ once the convective instabilities have travelled out of the domain.

At $R e \geqslant 110$ and $V_{r}=3$, the nonlinear flow becomes time-dependent and threedimensional. To ensure that this behaviour is independent of the outlet length, we have run also a three dimensional nonlinear simulation at $R e=110$ where the outlet length is increased from $L_{\text {out }}=40$ to $L_{\text {out }}=60$. The flow has the same nonlinear cycle as in the simulations with the shorter outlet length described below. In figure 16 we present the time evolution of the span-wise velocity for the case $R e=120$. Similar behaviour is observed for $R e \in[110-140]$. The nonlinear evolution of the flow displays a cyclic behaviour with a period of about 50 time units, where $t_{0}$ in the figure is the initial time of the cycle. The span-wise velocity first grows in time up to a critical threshold, where the maximum non dimensional spanwise velocity becomes $w \approx 0.85$ (see figure 16(a)). Above this threshold, the flow develops instabilities at the end of the large recirculation region, figure 16(b). These instabilities prevent the three-dimensional flow structurefrom saturating nonlinearly to a stationary state. Instead, they break down the structure and produce a convective packet traveling downstream, as shown in figure 16(c). Once 

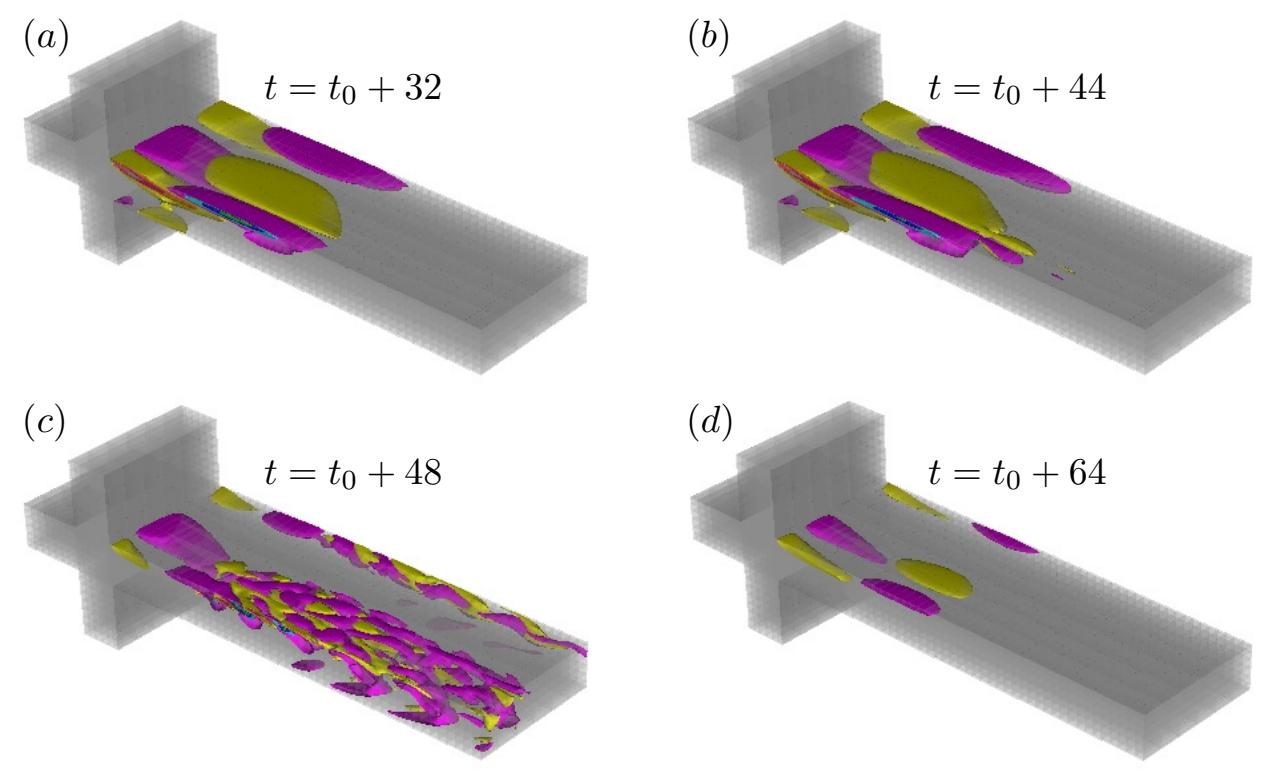

FiguRE 16. Time evolution of the span wise velocity at $R e=120$ and $V_{r}=3(a)$ initial amplification of velocity $(b)$ onset of instability at $w \approx 0.85(c)$ convective instabilities $(d)$ re-establishment of the span-wise velocity

the instabilities have been convected out of the domain, the flow returns to a nearly two-dimensional state, the span-wise velocity starts to grow again at the end of the re-circulation region, and the cycle repeats, figure $16(\mathrm{~d})$. The behaviour of the flow is similar at higher Reynolds number. In figure 17 we present the history of the $v$-velocity extracted from the probe signals for $R e=110$ and $R e=120$ when $V_{r}=3$. The cyclic behaviour of the nonlinear simulation is evident. Upstream, the velocity varies smoothly in time. Downstream, the flow shows rapid oscillations at the end of each cycle, which correspond to the secondary instability and the formation of the traveling packet. The period of each cycle for $R e=120$ is shorter and not constant in time with respect to the one for $R e=110$.

In summary, we observe a steady two-dimensional flow at $R e \leqslant 105$ and an unsteady three-dimensional final state at $R e \geqslant 110$. This behaviour is also shown in figure 6(b). The appearance of the nonlinear cycle is evident at $R e=110$ where the $v$-velocity at the centreline becomes time-dependent. This critical Reynolds number agrees qualitatively but not quantitatively with the results of the linear stability analysis, where the asymmetric base flow results in an unstable stationary global mode at $R e \geqslant 115$. DNS of the three-dimensional configuration with different initial conditions indicates that the second bifurcation is weakly subcritical. In the subcritical case the cycle is initiated by similar flow structures. The observed spanwise modulation growing at the beginning of the cycle (figure 16(a) for $R e=120$ ) resembles the leading global mode at the same Reynolds number. This is also true for $R e=110$. Hence, although the bifurcation is weakly subcritical, we assume that the physical mechanism behind the nonlinear cycle at $R e=110$ is the same as global instability at $R e>115$.

We further analyse the nonlinear cyclic behaviour of the flow by using the linearized Navier-Stokes solver on a three-dimensional base flow. We create an artificial base flow by adding the unstable span-wise dependent global mode to the two-dimensional base flow at $R e=120$. The amplitudes of the unstable mode are chosen to produce a maximum 
(a)

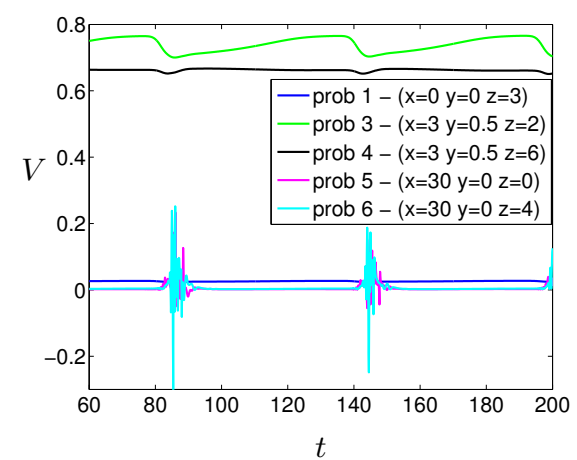

(b)

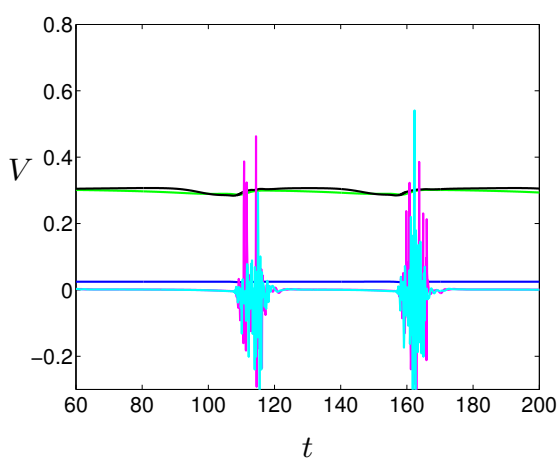

FigURE 17. $v$-velocity of the probe signal for $V_{r}=3$ and (a) $R e=110$, (b) $R e=120$

$(a)$

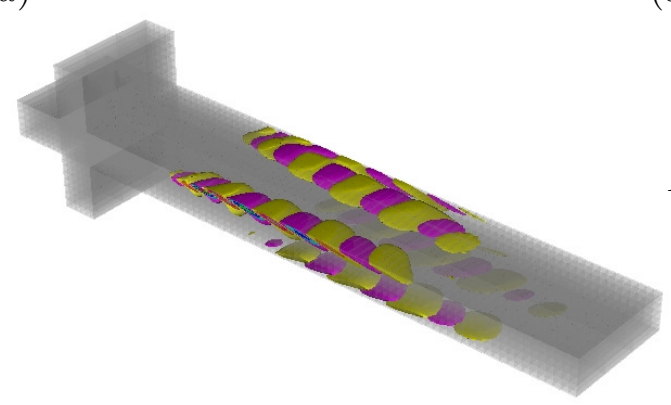

(b)

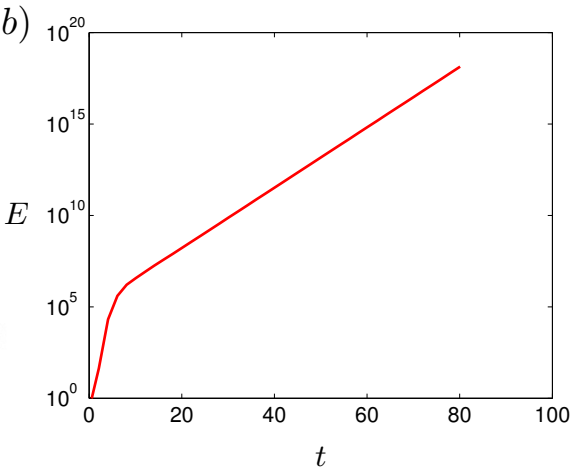

Figure 18. (a) Spatial structure and (b) exponential growth of the energy of the unstable traveling mode about the fictitious flow with $w=0.85$ at $R e=120$ and $V_{r}=3$

span-wise velocity of the new base flow in the range $0<w<1$. Marching the linearized Navier-Stokes solver about this fictitious flow, we observe, for $w \geqslant 0.85$, the appearance of an oscillatory mode that preserves its shape while growing exponentially in time. If the maximum of $w$ is less than 0.85 , the non-stationary mode eventually decays - i.e. the flow is stable. We depict the structure of the span-wise velocity component of the oscillatory mode in figure 18(a). It emerges at the end of the larger recirculation bubble and disappears downstream at a stream-wise location about 25 . The frequency of the oscillatory mode is about 1.25 which is close to the frequency of breakdown in the nonlinear simulation (about 1). Figure 18(b) presents the energy growth of the oscillatory mode. A significant transient growth of energy is evident for $0<t<5$, followed by exponential growth. The presence of this oscillatory unstable mode, which grows exponentially, reduces the span-wise modulation of the flow. Our hypothesis is that in the nonlinear DNS, this oscillatory mode leads to chaotic flow and nonlinear breakdown to a nearly two-dimensional flow at the end of each cycle. After breakdown, the span-wise modulation caused by the global mode $(\beta=1)$ starts to grow again and a new cycle starts.

\subsection{Sensitivity to wall suction}

The second bifurcation is caused by a three-dimensional stationary global mode around the asymmetric steady state. Here we examine the sensitivity of the second bifurcation to wall suction/blowing. 


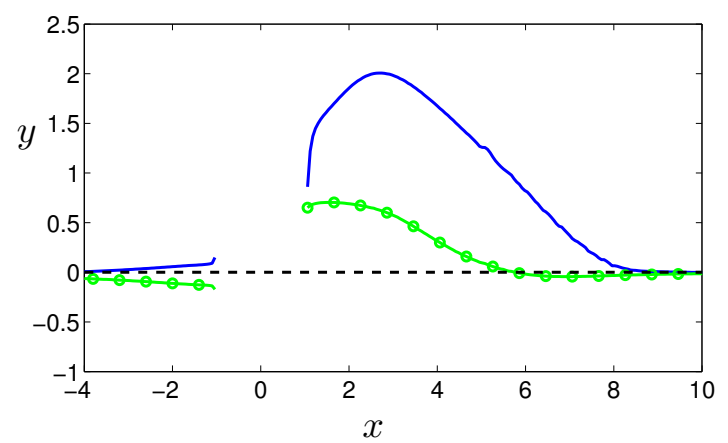

Figure 19. (Colour online) Sensitivity of the second bifurcation to wall suction $(\mathrm{Re}=115)$, at the adjacent wall of the largest bubble (solid blue line) and at the opposite wall (dotted green line)

The sensitivity to wall suction at both walls is shown in figure 19, for asymmetric flow at $R e=115$. Blowing away from the wall at the streamwise location of the two asymmetric recirculation bubbles is stabilizing, and suction destabilizing, as for the first bifurcation in $\S 4.2 .1$. The sensitivity at the second bifurcation is highest at the side of the larger recirculation bubble.

Some general observations about the control of the second bifurcation can be made. First of all, the magnitudes of the sensitivity to the wall suction are nearly twice as large as for the first bifurcation. This means that control of the second bifurcation is likely to be efficient. One should note that in order to obtain the best control effect, one would need to know at which wall the larger bubble is. Since the Pitchfork bifurcation might lead to a larger bubble on either wall, the control needs to be adjustable.

Stabilizing control of the second bifurcation at $R e=115$ is probably of limited interest because we have shown that the critical Reynolds number for the first bifurcation can be increased as far as $R e=150$ with control. However, destabilizing control of the second bifurcation could be interesting for inertial micromixers, for which a time-dependent state causes enhanced mixing.

\section{Conclusions}

In this paper, we have investigated the hydrodynamic instabilities of the flow in an X-junction using three-dimensional Direct Numerical Simulation (DNS), linear global stability, and sensitivity analysis.

For all the velocity ratios considered in this study, the $\mathrm{X}$-junction exhibits two bifurcations as the Reynolds number is increased. The destabilization first occurs through a pitchfork bifurcation to a two-dimensional, asymmetric steady state at $R e \approx 82.5$ for velocity ratio $V_{r}=3$. Global stability analysis around a symmetric flow at $\operatorname{Re}>82.5$ detects the zero-frequency unstable two-dimensional global mode, which is responsible for this first bifurcation. The core of the instability, both in terms of the structural sensitivity and the disturbance energy production, is localized symmetrically at the edges of the two recirculation bubbles. The kinetic energy budget of the perturbation is analysed, and the structure and amplitude of the lift-up term in the energy budget is shown to be almost identical to the total production. This together with the mode shapes, stationary streamwise elongated modes with strong streamwise velocity, shows that the lift-up mechanism causes the perturbation energy growth. This is similar to the finding of Lanzerstorfer \& Kuhlmann (2011) for the backwards-facing step, where at a certain parameter range, 
the vertical velocity perturbation extracts energy from the base flow shear to produce streamwise-elongated low and high speed streaks. The first bifurcation in the X-junction is however two-dimensional, as was the original lift-up mechanism identified by Landahl (1975). The presence of two recirculation bubbles make it possible to form antisymmetric modes, which induces a cross-stream pressure difference promoting asymmetry, and also minimises the dissipation by vertical velocity gradients.

We show that the shape of the recirculating region at the critical condition is unaffected by the velocity of the main channel and is determined only by the velocity of the side channels. Therefore, the value of the critical Reynolds number based on the velocity of the side channels is found to be almost constant for the range of velocity ratios studied. Our hypothesis is that the instability appears at a critical size of the recirculation zones (around 6 channel half widths for the first bifurcation), which are more affected by the side flows than by the main flow. Also in a previous study, an instability in a junction with different inflow-outflow structure appeared at the same length of the recirculation zone (Poole et al. 2014).

An adjustable control strategy for the first bifurcation is proposed in the form of distributed suction/blowing at the walls. This proves to be an efficient means to maintain a symmetric flow. In the sensitivity distribution, the positive values represent suction and the negative values represent blowing. This wall-normal velocity is then applied at both walls symmetrically in actual computations, at different small amplitudes, and the stability recalculated. The optimal stabilizing wall-normal velocity increases the critical Reynolds number for the first bifurcation from $R e=82.5$ to $R e>150$, with normalized amplitude of only $3 \%$ both in terms of maximum velocity and flow rate. The main stabilizing effect is due to blowing from the wall, which reduces both the backflow and the velocity gradient responsible for the lift-up mechanism.

The second bifurcation has more intricate dynamics. Linear stability of the steady asymmetric base flow, obtained after nonlinear saturation of the first instability, reveals that a new instability appears at $R e \approx 115$ for $V_{r}=3$, again as a zero-frequency global mode. This unstable mode has a span-wise wavenumber $\beta \approx 1$ for all velocity ratios $V_{r} \geqslant$ 1. As for the first bifurcation, the Reynolds number for the second bifurcation is dictated by the side channel velocities only or, physically, the size of the larger recirculation bubble. The structural sensitivity is localized in the middle of the bubble but the maximum perturbation kinetic energy is localized at the edge of the bubble. The lift up term of the production of the perturbation kinetic energy is dominating and its structure is similar to the total production. This suggests that the second bifurcation is also due to the lift-up instability mechanism similar to the findings of Marquet et al. (2009) and Lanzerstorfer \& Kuhlmann (2011).

The behaviour at the second bifurcation is clearly defined by means of non-linear simulations. These three-dimensional simulations show that the flow is steady and twodimensional, regardless of the initial condition, for $R e \leqslant 105$. However, for $R e \geqslant 110$, we observe a nonlinear cycle: a two-dimensional asymmetric flow, the growth of a spanwise modulation, a time-dependent chaotic flow, traveling wave pocket and return to a nearly two-dimensional asymmetric flow. We use the linearized Navier-Stokes solver to explain the nonlinear breakdown of the flow by a secondary instability: we identify an oscillatory unstable mode that grows exponentially about an artificial base flow, constructed by combining the steady asymmetric base flow with the unstable three-dimensional steady mode, when the amplitude of the spanwise modulation reaches a critical value. The frequency of the oscillatory unstable mode is found to be similar to the one of the nonlinear breakdown. The DNS indicates that the second bifurcation is weakly subcritical because it occurs at $R e=110$, while a three-dimensional global mode destabilizes first 
at $R e=115$. However, the structure of the growing spanwise modulation resembles the leading global mode (at $R e=110$, it is marginally stable), and we conclude that the same physical mechanism is active for $R e \geqslant 110$. The sketch representing the bifurcations versus the Reynolds number for the flow with velocity ratio $V_{r}=3$ is presented in figure 6 (b).

Finally, we show that the sensitivity to wall suction at the second bifurcation is twice as large as at the first bifurcation - i.e. that this control is efficient. Given that the critical Reynolds number for the first bifurcation can be increased as far as $R e=150$ by boundary blowing/suction, stabilization of the second bifurcation should be studied at higher Reynolds number. Destabilizatin of the second bifurcation with control could be useful in order to trigger instabilities that increase mixing in micromixers.

\section{Acknowledgment}

We would like to especially thank Hugh Blackburn for providing us with the linear stability solver, DOG. We also thank Karl Håkansson, Daniel Söderberg and Fredrik Lundell for discussions about the flow geometry and Flavio Giannetti for his comments and suggestions. This work was performed on the computational facilities provided by SNIC (Swedish National Infrastructure for Computing), and the Darwin Supercomputer of the University of Cambridge High Performance Computing Service (http://www.hpc.cam.ac.uk/). The financial support for Tammisola and Juniper from the European Research Council through Project ALORS 2590620 is gratefully acknowledged. Travel support for Tammisola was provided by Wallenberg Wood Science Center, Sweden.

\section{REFERENCES}

Albensoeder, S., Kuhlmann, H. C. \& Rath, H. J. 2001 Three-dimensional centrifugal-flow instabilities in the lid-driven-cavity problem. Physics of Fluids 13, 121.

Barkley, D., Blackburn, H. M. \& Sherwin, S. J. 2008 Direct optimal growth analysis for timesteppers. Int. J. Numer. Meth. Fluids. 57, 1435-1458.

Barkley, D., Gomes, G. M. \& Henderson, R. D. 2002 Three-dimensional instability in flow over a backward-facing step. J. Fluid Mech. 473, 167-190.

BEst, J. L. 1987 Flow dynamics at river channel confluences: implications for sediment transport and bed morphology. Recent developments of fluvial sedimentology (by The society of Economic Paleontologists and Mineralogists SEPM) p. 2735.

Best, J. L. \& REID, I. 1984 Separation zone at open-channel junctions. Journal of Hydraulic Engineering 110, 15881594.

Bourque, C. \& Newman, B. G. 1960 Reattachment of a two-dimensional, incompressible jet to an adjacent flat plate. Aeronaut Quart 11, 201-232.

BRAnDT, L. 2014 The lift-up effect: The linear mechanism behind transition and turbulence in shear flows. European Journal of Mechanics - B/Fluids ((doi: 10.1016/j.euromechflu.2014.03.005)).

Cherdron, W., Durst, F. \& Whitelaw, J. H. 1978 Asymmetric flows and instabilities in symmetric ducts with sudden expansion. J. Fluid Mech. 84, 13-31.

Chiang, T. \& Sheu, T. W. H. 2002 Bifurcations of flow through plane symmetric channel contraction. Journal of Fluids Engineering 124, 444451.

Chomaz, J. M. 2005 Global instabilities in spatially developing flows: non-normality and nonlinearity. Annu. Rev. Fluid Mech. 37, 357-392.

Fani, A., Camarri, S. \& Salvetti, M. V. 2012 Stability analysis and control of the flow in a symmetric channel with a sudden expansion. Physics of Fluids 24 (084102).

Fani, A., Camarri, S. \& Salvetti, M. V. 2013 Investigation of the steady engulfment regime in a three-dimensional t-mixer. Physics of Fluids 25 (064102). 
Fearn, R. M., Mullin, T. \& Cliffe, K. A. 1990 Nonlinear flow phenomena in a symmetric sudden expansion. J. Fluid Mech. 211, 595-608.

Gallaire, F., Marquillie, M. \& Ehrenstein, U. 2007 Three-dimensional transverse instabilities in detached boundary layer. J. Fluid Mech. 571, 221-223.

Giannetti, F. \& Luchini, P. 2007 Structural sensitivity of the first instability of the cylinder wake. J. Fluid Mech. 581, 167-197.

HÅKANSSON, K. 2012 Orientation of elongated particles in shear and extensional flow. Licentiate thesis in Engineering Mechanics Stockholm, Sweden .

Haque, S., Lashgari, I., Giannetti, F. \& Brandt, L. 2012 Stability of fluids with sheardependent viscosity in the lid-driven cavity. J. Non-Newtonian Fluid Mech. 173, 49-61.

HiLl, D. C. 1992 A theoretical approach for analyzing the re-stabilization of wake. AIAA Paper pp. 92-0067.

Joanicot, M. \& Ajdari, A. 2005 Droplet control for microfluidics. Science 309, 887888.

Kinahan, M. . E., Filippidi, E., Köster, S., Hu, X., Evans, H. M., Pfohl, T., Kaplan, D. L. \& J., W. 2011 Tunable silk: Using microfluidics to fabricate silk fibers with controllable properties. Biomacromolecules 12, 15041511.

Kockmann, N., Foll, C. \& Woias, P. 2003 Flow regimes and mass transfer characteristics in static micromixers. Proc. SPIE 4982, 319-329.

LANDAhL, M. T. 1975 Dynamics and control of global instabilities in open flows: a linearized approach. SIAM J. Appl. Maths 28 (735-756).

Lanzerstorfer, D. \& Kuhlmann, H. 2011 Global stability of the two-dimensional flow over a backward-facing step. Journal of Fluid Mechanics 693, 127.

Lanzerstorfer, D. \& Kuhlmann, H. C. 2012 Global stability of multiple solutions in plane sudden-expansion flow. Journal of Fluid Mechanics 702, 378-402.

Luchini, P. \& Bottaro, A. 2014 Adjoint equations in stability analysis. Annu. Rev. Fluid Mech. 46, 493-517.

Maday, Y. \& PAterA, A. T. 1989 Spectral element methods for the navier-stokes equations. State-of-the-art surveys in computational mechanics, ASME (ed. A.K. Noor) 18, 71143.

Marquet, O., Lombardi, M., Chomaz, J. M., Sipp, D. \& Jacquin, L. 2009 Direct and adjoint global modes of a recirculation bubble: lift-up and convective non-normalities. $J$. Fluid Mech. 622, 1-21.

Marquet, O. \& Sipp, D. 2010 Active, steady control of vortex shedding: an adjoint-based sensitivity approach. Seventh IUTAM Symposium on Laminar-Turbulent Transition IUTAM Bookseries 18, 259-264.

Marquet, O., Sipp, D. \& Jacquin, L. 2008 Sensitivity analysis and passive control of cylinder flow. J. Fluid Mech. 615, 221-252.

Mizushima, J. \& Shiotani, Y. 2000 Transitions and instabilities of flow in a symmetric channel with a suddenly expanded and contracted part. Journal of Fluid Mechanics 434, 355369.

NGuyen, N. G. \& Wu, Z. 2005 Micromixers a review. Journal of Micromechanics and Microengineering 15.

Oliveira, M. S. N., Pinho, F. T. \& Alves, M. A. 2012 Divergent streamlines and free vortices in Newtonian fluid flows in microfluidic flow-focusing devices. J. Fluid Mech. 711, 171-191.

Passaggia, P. Y., Leweke, T. \& Ehrenstein, U. 2012 Transverse instability and lowfrequency flapping in incompressible separated boundary layer flows: an experimental study. J. Fluid Mech. 703, 363-373.

PATERA, A. T. 1984 A spectral element method for fluid dynamics: Laminar flow in a channel expansion. J. Computational Physics 54, 468-488.

Pironneau, O., Hecht, F. \& Morice, J. 2013 Freefem++. Web page. Http://www.freefem.org. .

Poole, R. J., Alves, M. A. \& Oliveira, P. J. 2007 Purely elastic flow asymmetries. Phys. Rev. Lett. 99, 164503.

Poole, R. J., Rocha, G. N. \& Oliveira, P. J. 2014 A symmetry-breaking inertial bifurcation in a cross-slot flow. Computers and Fluids 93, 164503.

Pralits, J. O., Brandt, L. \& Giannetti, F. 2010 Instability and sensitivity of the flow around a rotating circular cylinder. J. Fluid Mech. 650, 513-536.

Rodriguez, D. \& Theofilis, V. 2010 Structural changes of laminar separation bubbles induced by global linear instability. J. Fluid Mech. 655, 280-305. 
Shabayek, S., Steffler, P. \& Hicks, F. 2002 Dynamic model for subcritical combining flows in channel junctions. Journal of Hydraulic Engineering 128, 821828.

Sherwin, S. J. \& Blackburn, H. M. 2005 Three-dimensional instabilities and transition of steady and pulsatile axisymmetric stenotic flows. J. Fluid Mech. 533, 297-327.

Sipp, D., Marquet, O., Meliga, P. \& Barbagallo, A. 2010 Dynamics and control of global instabilities in open flows: a linearized approach. Appl. Mech. Reviews 63 (030801).

Sobey, I. J. \& Drazin, P. G. 1986 Bifurcations of the two-dimensional channel flows. J. Fluid Mech. 171, 263-287.

Tomas, S., Ameel, T. \& Guilkey, J. 2010 Mixing kinematics of moderate Reynolds number flows in a T-channel. Physics of Fluids 22, 013601.

Tufo, H. M. \& Fischer, P. F. 1999 Terascale spectral element algorithms and implementations. Supercomputing, ACM/IEEE Conference p. 68. 